\title{
On the interactions of vorticity, divergence and deformation in a meso-a-scale vortex
}

\author{
$\mathrm{Na} \mathrm{Li}^{1,2}$ - Lingkun $\operatorname{Ran}^{1} \cdot$ Shouting Gao ${ }^{1}$
}

Received: 13 April 2018 / Accepted: 21 May 2019 / Published online: 19 July 2019

(c) The Author(s) 2019

\begin{abstract}
Vorticity, divergence and deformation are three differentiation properties of the air flow. In this paper, by deriving the tendency equations of squared vorticity (SV), squared divergence (SD) and squared deformation (SE), it is found that the three properties can interact with each other through the terms with opposite signs in the equations. These opposite-sign terms permit a quantitative discussion on the interactions between vorticity, divergence and deformation, and the role of these interactions on weather developments. The corresponding framework is first applied to investigate the development of an extratropical meso- $\alpha$-scale cyclonic vortex in China. Through diagnosing the SV equation and the interactive terms in the vortex event, it is found that the interaction between SV and SD was the controlling factor for the evolution of the vortex. The transition of SD-SV can intensify the vortex, while the transition of SV-SD causes the vortex to die out. This is consistent with the relation between vorticity and divergence that has been obtained from the vertical vorticity equation. Apart from this, a new finding is that deformation also plays a role in influencing the vortex development. On one hand, deformation can interact with vorticity to affect the vortex directly. On the other hand, deformation interacts with divergence and influence the vortex development in an indirect way. These results provide a new insight on the vortex development which is worth further investigating in the future.
\end{abstract}

\section{Introduction}

Research on winds has been central to learning about the rules governing the evolution of weather systems, which is regarded as fundamental for the advancement of weather forecasting. Taking the first two terms in a Taylor's series expansion of the horizontal wind field $(u, v)$ about a fixed point $\left(x_{0}, y_{0}\right)$ obtains (Petterssen 1956; Dutton 1976; Bluestein 1992; Gao 2007)

Responsible Editor: C. Simmer.

Electronic supplementary material The online version of this article (https://doi.org/10.1007/s00703-019-00674-9) contains supplementary material, which is available to authorized users.

$\mathrm{Na} \mathrm{Li}$

lina@mail.iap.ac.cn

1 Key Laboratory of Cloud-Precipitation Physics and Severe Storms (LACS), Institute of Atmospheric Physics, Chinese Academy of Sciences, Beijing 100029, China

2 Plateau Atmosphere and Environment Key Laboratory of Sichuan Province, Sichuan 610103, China

$$
\left(\begin{array}{c}
u \\
v
\end{array}\right)_{x, y}=\left(\begin{array}{l}
u \\
v
\end{array}\right)_{x_{0}, y_{0}}+\left(\begin{array}{ll}
\partial u / \partial x & \partial u / \partial y \\
\partial v / \partial x & \partial v / \partial y
\end{array}\right)_{x_{0}, y_{0}}\left(\begin{array}{c}
x-x_{0} \\
y-y_{0}
\end{array}\right)
$$

In Eq. (1), the first term represents a rigid body translation. The second term, which is associated with the gradient of the displacement, gives the shape change of the horizontal wind and is referred to as velocity gradient tensor (e.g. Schielicke et al. 2016). The velocity gradient tensor can be separated into several parts:

$\left(\begin{array}{c}u \\ v\end{array}\right)_{x, y}=\left(\begin{array}{c}u \\ v\end{array}\right)_{x_{0}, y_{0}}+\frac{1}{2}\left(\begin{array}{cc}D+E_{\mathrm{st}} & E_{\mathrm{sh}}-\zeta \\ \zeta+E_{\mathrm{sh}} & D-E_{\mathrm{st}}\end{array}\right)_{x_{0}, y_{0}}\left(\begin{array}{c}x-x_{0} \\ y-y_{0}\end{array}\right)$,

where $\zeta=\frac{\partial v}{\partial x}-\frac{\partial u}{\partial y}$ is the vertical vorticity, $D=\frac{\partial u}{\partial x}+\frac{\partial v}{\partial y}$ is the divergence, and $E_{\mathrm{sh}}=\frac{\partial v}{\partial x}+\frac{\partial u}{\partial y}$ and $E_{\mathrm{st}}=\frac{\partial u}{\partial x}-\frac{\partial v}{\partial y}$ are the shear and stretching deformation, respectively. Unlike vorticity or divergence, the shearing deformation and stretching deformation are not invariant under coordinate transformations. The total deformation $E\left(E=\sqrt{E_{\mathrm{sh}}^{2}+E_{\mathrm{st}}^{2}}\right)$, however, is invariant (Bluestein 1992). The vorticity, divergence and deformation, respectively, describe the changes of the angle, 
area and shape of the continuous atmosphere, denoting the differentiation properties of the motion. These different properties of motion bear significant relations with the evolution of weather systems, as demonstrated by numerous observational and theoretical studies.

The vorticity, apart from being used directly to measure and identify the vortex system (Sadarjoen and Post 2000; Hoskins and Hodges 2002; Luo and Dai 2008), is also involved in the potential vorticity theorem to explain the dynamics of cyclogenesis, the interactions between the upper-level and lower-level atmosphere, tropical cyclogenesis and the long-term maintenance of mesoscale convective systems (MCSs) (Danielsen 1968; Hoskins et al. 1985; Haynes and McIntyre 1987; Raymond and Jiang 1990; Davis and Emanuel 1991; Montgomery and Enagonio 1998), barotropic instability (Mak and Cai 1989), and the turbulence dynamics due to the conservation property of vorticity in a barotropic non-divergent environment (Okubo 1970; Weiss 1991; Rozoff et al. 2006; Wang 2008). The divergence, based on the continuity equation, seems highly relevant for the phenomena with vertical motion, such as the initiation or intensification of convection due to moisture accumulation, the pumping effect, or passage of gravity waves (Uccellini and Koch 1987; Ziegler et al. 1997; Kalthoff et al. 2009; Ueno et al. 2009; Masunaga 2013). In addition, it is also a basis for nonlinear balance theory, which is often used to study the inner dynamics of the evolution of MCSs or the diagnosis of the existence of gravity waves (Raymond and Jiang 1990; Olsson and Cotton 1997; Zhang 2004; Raymond 2006). Compared to the vorticity and divergence, the effects of deformation on the development of weather systems are less frequently considered and applied. In atmospheric dynamics, deformation is mainly related to frontogenesis according to the well-known theorem that deformation drives frontogenesis when the angle between the dilatation axis and the isentropes is less than $45^{\circ}$ (Petterssen 1956; Stone 1966; Keyser and Pecnick 1985; Keyser et al. 1988). Mak and Cai (1989) and Cai (1992) discussed the stability of the disturbance in the deformation field and found that a perturbation must be properly configured with the deformation field to optimally extract (kinetic) energy from the basic flow. Spensberger and Spengler (2014) reveals that deformation is also related to the movement and evolution of the upper-level jet streams, the orographic blocking and Rossby wave breaking through a climatology study of deformation.

While most previous studies have paid attention to the separate effects of vorticity, divergence and deformation on the evolution of weather systems, the overall roles of the three factors combined are less well studied and remain more elusive. A single quantity (vorticity, divergence, or deformation) only describes portion of the flow features, but cannot reflect the holistic structure of the flow. The vorticity, divergence and deformation of different proportions would result in different flow patterns, which then endure development of different weathers. In fact, it can be anticipated that change in one property of motion (e.g. divergence) would also cause change in other properties of motion (e.g. vorticity or deformation), considering that these three properties are differentials of the same motion. These changes would affect the structure of the flow and then weather evolutions. It is thus natural to question how the different properties of motion interact with one another and what roles these interactions play in the evolution of weather systems. On this topic, there has already been some literature concerning the interaction between vorticity and divergence. With the aid of the equations for vorticity and divergence [e.g. Eqs. (6) and (19) in Stevens (1979) and Eqs. (6.72) and (6.76) in Lv et al. (2008)], one can find vorticity involves divergence on the right-hand side of its tendency equation, while divergence also involves vorticity in its forcing term of the tendency equation. This means that, on the one hand, divergence would influence the evolution of vorticity, while on the other hand, a change in vorticity would further impact on the evolution of divergence, which is actually a feedback process (Wang and Sun 1988). However, no evident interactive terms appear in the vorticity and divergence equations, which limit a quantitative measure of the vorticity-divergence interaction and its impact on weather systems. Moreover, apart from the vorticity-divergence interaction, whether or not there is interaction between deformation and vorticity, or between deformation and divergence, is not clear. If there is, what impacts these interactions have on weather systems is not clear as well. These scientific questions can be summarized as follows: (1) Does the deformation interact with the other two properties-vorticity and divergence? (2) Can the interactions of the three properties be quantitatively measured and discussed? (3) What roles have the interactions of the three properties played in the weather systems' evolutions?

The purpose of this paper is just to answer the above questions. In Sect. 2, we introduce the squared vorticity (SV), squared divergence (SD), and squared deformation (SE) to measure the intensity of rotation, divergence and deformation. By deriving the tendency equations of these three quantities, the opposite-sign terms (terms with same absolute values but opposite signs) are identified as the interaction terms of vorticity, divergence and deformation. In Sect. 3, the above framework is applied during the lifecycle of a meso- $\alpha$ vortex in China, with the interactions of vorticity, divergence and deformation and their roles on the development of the vortex discussed. Finally, we summarize the key findings of the study and give the discussions in Sect. 4. 


\section{Formulas and methodology}

When discussing the changes of some variables, absolute values or the square of these variables are usually involved instead of the variables themselves due to their signs which tend to make the discussion complicated. For example, positive values of the individual change of vorticity $\left(\frac{\mathrm{d} \zeta}{\mathrm{d} t}>0\right)$ not only represent the increase of cyclonic vorticity, but may also indicate the decrease of anticyclonic vorticity. The squared vorticity can nevertheless better represent the intensity change of the rotation. In addition, as stated in the introduction, the shear deformation $E_{\mathrm{sh}}$ and stretching deformation $E_{\mathrm{st}}$ change with the coordinate. Therefore, the total deformation, $E$, which is the square root of the sum of the shearing deformation square and stretching deformation square, is usually used to measure the intensity of the deformation. It is much easier to discuss the deformation square than the deformation itself. Due to these two reasons, we use the squared forms of vorticity, divergence and deformation to do the discussions of interactions between them, which are defined as follows:

$\varepsilon=\frac{1}{2} \zeta^{2}=\frac{1}{2}\left(\frac{\partial v}{\partial x}\right)^{2}+\frac{1}{2}\left(\frac{\partial u}{\partial y}\right)^{2}-\frac{\partial v}{\partial x} \frac{\partial u}{\partial y}$

$\delta=\frac{1}{2} D^{2}=\frac{1}{2}\left(\frac{\partial u}{\partial x}\right)^{2}+\frac{1}{2}\left(\frac{\partial v}{\partial y}\right)^{2}+\frac{\partial u}{\partial x} \frac{\partial v}{\partial y}$

$\sigma=\frac{1}{2} E^{2}=\frac{1}{2}\left(E_{s h}^{2}+E_{s t}^{2}\right)=\frac{1}{2}\left(\frac{\partial v}{\partial x}\right)^{2}+\frac{1}{2}\left(\frac{\partial u}{\partial y}\right)^{2}+\frac{\partial v}{\partial x} \frac{\partial u}{\partial y}+\frac{1}{2}\left(\frac{\partial u}{\partial x}\right)^{2}+\frac{1}{2}\left(\frac{\partial v}{\partial y}\right)^{2}-\frac{\partial u}{\partial x} \frac{\partial v}{\partial y}$.

In Eqs. (3)-(5), $\varepsilon$ is half the squared vorticity (SV), $\delta$ is half the squared divergence (SD), and $\sigma$ is half the squared deformation (SE). $\sigma$ is composed by half the squared shearing deformation $\left(\mathrm{SE}_{\mathrm{R}}, \frac{1}{2} E_{s h}^{2}=\frac{1}{2}\left(\frac{\partial v}{\partial x}\right)^{2}+\frac{1}{2}\left(\frac{\partial u}{\partial y}\right)^{2}+\frac{\partial v}{\partial x} \frac{\partial u}{\partial y}\right)$ and half the squared stretching deforma$\operatorname{tion}\left(\mathrm{SE}_{\mathrm{S}}, \frac{1}{2} E_{s t}^{2}=\frac{1}{2}\left(\frac{\partial u}{\partial x}\right)^{2}+\frac{1}{2}\left(\frac{\partial v}{\partial y}\right)^{2}-\frac{\partial u}{\partial x} \frac{\partial v}{\partial y}\right)$. Here, the "half" operation is to make the following interactive terms simpler, which will not change the analysis results. The three squared quantities represent the intensity of the rotation, divergence and deformation, respectively.

In $p$-coordinates, the basic horizontal motion equations of the atmosphere on an $f$ plane without friction can be written as

$\frac{\partial u}{\partial t}+\mathbf{V} \cdot \nabla u=f v-g \frac{\partial z}{\partial x}$,

$\frac{\partial v}{\partial t}+\mathbf{V} \cdot \nabla v=-f u-g \frac{\partial z}{\partial y}$, where $\mathbf{V}=(u, v, \omega)$ is the three-dimensional velocity vector, $z$ geopotential height, $g$ gravitational acceleration, and $f$ is the Coriolis parameter, which is a constant here. Taking the partial derivatives of Eqs. (3)-(5) to $t$ obtains

$\frac{\partial \varepsilon}{\partial t}=\zeta \frac{\partial \zeta}{\partial t}=\zeta\left[\frac{\partial}{\partial x}\left(\frac{\partial v}{\partial t}\right)-\frac{\partial}{\partial y}\left(\frac{\partial u}{\partial t}\right)\right]$,

$\frac{\partial \delta}{\partial t}=D \frac{\partial D}{\partial t}=D\left[\frac{\partial}{\partial x}\left(\frac{\partial u}{\partial t}\right)+\frac{\partial}{\partial y}\left(\frac{\partial v}{\partial t}\right)\right]$,

$$
\begin{aligned}
\frac{\partial \sigma}{\partial t}=E_{\mathrm{sh}} \frac{\partial E_{\mathrm{sh}}}{\partial t}+E_{\mathrm{st}} \frac{\partial}{\partial t} E_{\mathrm{st}}= & E_{\mathrm{sh}}\left[\frac{\partial}{\partial x}\left(\frac{\partial v}{\partial t}\right)+\frac{\partial}{\partial y}\left(\frac{\partial u}{\partial t}\right)\right] \\
& +E_{\mathrm{st}}\left[\frac{\partial}{\partial x}\left(\frac{\partial u}{\partial t}\right)-\frac{\partial}{\partial y}\left(\frac{\partial v}{\partial t}\right)\right] .
\end{aligned}
$$

Substituting Eqs. (6) and (7) into Eqs. (8)-(10) obtains the tendency equations of SV, SD, and SE:

$\frac{\partial \varepsilon}{\partial t}=-\mathbf{V} \cdot \nabla \varepsilon-f \zeta D-\zeta^{2} D+\zeta\left(\frac{\partial \omega}{\partial y} \frac{\partial u}{\partial p}-\frac{\partial \omega}{\partial x} \frac{\partial v}{\partial p}\right)$,

$$
\begin{aligned}
\frac{\partial \delta}{\partial t}= & -\mathbf{V} \cdot \nabla \delta-D\left(\frac{\partial u}{\partial x}\right)^{2}-D\left(\frac{\partial v}{\partial y}\right)^{2}-2 \frac{\partial v}{\partial x} \frac{\partial u}{\partial y} D \\
& -D\left(\frac{\partial \omega}{\partial x} \frac{\partial u}{\partial p}+\frac{\partial \omega}{\partial y} \frac{\partial v}{\partial p}\right)+f \zeta D-D g \nabla_{h}^{2} z
\end{aligned}
$$

$$
\begin{aligned}
\frac{\partial \sigma}{\partial t}= & -\mathbf{V} \cdot \nabla \sigma-E^{2} D-E_{\mathrm{sh}}\left(\frac{\partial \omega}{\partial y} \frac{\partial u}{\partial p}+\frac{\partial \omega}{\partial x} \frac{\partial v}{\partial p}\right) \\
& -E_{\mathrm{st}}\left(\frac{\partial \omega}{\partial x} \frac{\partial u}{\partial p}-\frac{\partial \omega}{\partial y} \frac{\partial v}{\partial p}\right) \\
& -2 E_{\mathrm{sh}} g \frac{\partial^{2} z}{\partial x \partial y}-E_{\mathrm{st}} g \frac{\partial^{2} z}{\partial x^{2}}+E_{\mathrm{st}} g \frac{\partial^{2} z}{\partial y^{2}} .
\end{aligned}
$$

To discuss the interactions of vorticity, divergence and deformation, the terms with same absolute values but opposite signs (denoted as "opposite-sign terms" in the following) of Eqs. (11)-(13), which may be sources or sinks of the corresponding quantity, are identified as their interaction terms. Take the advection term as an example, the advection terms in Eqs. (11) and (13) can, respectively, be written as

$$
\begin{aligned}
-\mathbf{V} \cdot \nabla \varepsilon & =-\mathbf{V} \cdot \nabla\left[\frac{1}{2}\left(\frac{\partial v}{\partial x}-\frac{\partial u}{\partial y}\right)^{2}\right] \\
& =-\mathbf{V} \cdot \nabla\left[\frac{1}{2}\left(\frac{\partial v}{\partial x}\right)^{2}+\frac{1}{2}\left(\frac{\partial u}{\partial y}\right)^{2}\right]+\mathbf{V} \cdot \nabla\left(\frac{\partial v}{\partial x} \frac{\partial u}{\partial y}\right),
\end{aligned}
$$




$$
\begin{aligned}
-\mathbf{V} \cdot \nabla \sigma= & -\mathbf{V} \cdot \nabla\left[\frac{1}{2}\left(E_{s h}^{2}+E_{s t}^{2}\right)\right] \\
= & -\mathbf{V} \cdot \nabla\left[\frac{1}{2}\left(\frac{\partial v}{\partial x}\right)^{2}+\frac{1}{2}\left(\frac{\partial u}{\partial y}\right)^{2}+\frac{1}{2}\left(\frac{\partial u}{\partial x}\right)^{2}\right. \\
& \left.+\frac{1}{2}\left(\frac{\partial v}{\partial y}\right)^{2}-\frac{\partial u}{\partial x} \frac{\partial v}{\partial y}\right]-\mathbf{V} \cdot \nabla\left(\frac{\partial v}{\partial x} \frac{\partial u}{\partial y}\right)
\end{aligned}
$$

Then, the opposite-sign term between the SV and SE equations associated with the advection term can be easily identified by comparing Eqs. (14) and (15), which is $\mathbf{V} \cdot \nabla\left(\frac{\partial v}{\partial x} \frac{\partial u}{\partial y}\right)$. However, it is important to note whether or not this opposite-sign term and the residual terms in each equation can be cancelled out. To explain this, decompose the total wind into three parts (Bishop 1996a, b; Cao et al. 2014):

$\mathbf{V}=\mathbf{V}_{1}+\mathbf{V}_{2}+\mathbf{V}_{h}$,

where $\mathbf{V}_{1}=\left(u_{1}, v_{1}\right)$ is the purely rotational wind, which satisfies $\mathbf{V}_{1}=-\nabla \times(\psi \mathbf{k})$ and $D_{\psi}=0 ; \mathbf{V}_{2}=\left(u_{2}, v_{2}\right)$ is the purely divergent wind, which has $\mathbf{V}_{2}=\nabla_{h} \chi$ and $\zeta_{\chi}=0$; and $\mathbf{V}_{h}=\left(u_{h}, v_{h}\right)$ is the harmonic environmental wind, which is non-rotational and non-divergent according to Bishop (1996a) and Cao et al. (2014). Taking Eq. (16) into Eqs. (14) and (15), the advection terms can be rewritten as

$$
\begin{aligned}
-\mathbf{V} \cdot \nabla \sigma= & -\mathbf{V} \cdot \nabla\left[\frac{1}{2}\left(\frac{\partial v}{\partial x}\right)^{2}+\frac{1}{2}\left(\frac{\partial u}{\partial y}\right)^{2}+\frac{1}{2}\left(\frac{\partial u}{\partial x}\right)^{2}\right. \\
& \left.+\frac{1}{2}\left(\frac{\partial v}{\partial y}\right)^{2}-\frac{\partial u}{\partial x} \frac{\partial v}{\partial y}\right] \\
& -\mathbf{V} \cdot \nabla\left(\frac{\partial v_{1}}{\partial x} \frac{\partial u_{1}}{\partial y}\right)-\mathbf{V} \cdot \nabla\left(\frac{\partial v_{1}}{\partial x} \frac{\partial\left(u_{2}+u_{h}\right)}{\partial y}\right) \\
& -\mathbf{V} \cdot \nabla\left(\frac{\partial\left(v_{2}+v_{h}\right)}{\partial x} \frac{\partial u}{\partial y}\right)
\end{aligned}
$$

According to Eqs. (17) and (18), the interaction term associated with the advection between SV equation and SE equation becomes $\mathbf{V} \cdot \nabla\left(\frac{\partial v_{1}}{\partial x} \frac{\partial u_{1}}{\partial y}\right)$, which is considered to be more simpler than $\mathbf{V} \cdot \nabla\left(\frac{\partial v}{\partial x} \frac{\partial u}{\partial y}\right)$. So, in the following derivations, the total wind on the right-hand side of Eqs. (11)-(13) is first decomposed before identifying the opposite-sign terms between each other. Here, the method used to decompose the wind comes from Cao et al. (2014) in which the piecewise continuous integration numerical scheme (Cao and $\mathrm{Xu}$ 2011) is used to solve $\mathbf{V}_{1}$ and $\mathbf{V}_{2}$, and the iterative method from Chen and Kuo (1992a) is adopted to solve for $\mathbf{V}_{h}$.

$$
\begin{aligned}
& -\mathbf{V} \cdot \nabla \varepsilon=-\mathbf{V} \cdot \nabla\left[\frac{1}{2}\left(\frac{\partial v_{1}}{\partial x}+\frac{\partial\left(v_{2}+v_{h}\right)}{\partial x}\right)^{2}+\frac{1}{2}\left(\frac{\partial u_{1}}{\partial y}+\frac{\partial\left(u_{2}+u_{h}\right)}{\partial y}\right)^{2}\right] \\
& +\mathbf{V} \cdot \nabla\left[\left(\frac{\partial v_{1}}{\partial x}+\frac{\partial\left(v_{2}+v_{h}\right)}{\partial x}\right)\left(\frac{\partial u_{1}}{\partial y}+\frac{\partial\left(u_{2}+u_{h}\right)}{\partial y}\right)\right]=-\mathbf{V} \cdot \nabla\left[\frac{1}{2}\left(\frac{\partial v_{1}}{\partial x}\right)^{2}+\frac{1}{2}\left(\frac{\partial u_{1}}{\partial y}\right)^{2}\right] \\
& -\mathbf{V} \cdot \nabla\left[\frac{1}{2}\left(\frac{\partial\left(v_{2}+v_{h}\right)}{\partial x}\right)^{2}+\frac{\partial v_{1}}{\partial x} \frac{\partial\left(v_{2}+v_{h}\right)}{\partial x}+\frac{1}{2}\left(\frac{\partial\left(u_{2}+u_{h}\right)}{\partial y}\right)^{2}+\frac{\partial u_{1}}{\partial y} \frac{\partial\left(u_{2}+u_{h}\right)}{\partial y}\right] \\
& +\mathbf{V} \cdot \nabla\left(\frac{\partial v_{1}}{\partial x} \frac{\partial u_{1}}{\partial y}\right)+\mathbf{V} \cdot \nabla\left[\frac{\partial v_{1}}{\partial x} \frac{\partial\left(u_{2}+u_{h}\right)}{\partial y}+\frac{\partial\left(v_{2}+v_{h}\right)}{\partial x} \frac{\partial u_{1}}{\partial y}+\frac{\partial\left(v_{2}+v_{h}\right)}{\partial x} \frac{\partial\left(u_{2}+u_{h}\right)}{\partial y}\right] \\
& =-\mathbf{V} \cdot \nabla\left[\frac{1}{2}\left(\frac{\partial v_{1}}{\partial x}\right)^{2}+\frac{1}{2}\left(\frac{\partial u_{1}}{\partial y}\right)^{2}\right]+\mathbf{V} \cdot \nabla\left(\frac{\partial v_{1}}{\partial x} \frac{\partial u_{1}}{\partial y}\right) \\
& -\mathbf{V} \cdot \nabla\left[\frac{1}{2}\left(\frac{\partial v_{2}+v_{h}}{\partial x}-\frac{\partial u_{2}+u_{h}}{\partial y}\right)^{2}+\frac{\partial v_{1}}{\partial x}\left(\frac{\partial v_{2}+v_{h}}{\partial x}-\frac{\partial u_{2}+u_{h}}{\partial y}\right)+\frac{\partial u_{1}}{\partial y}\left(\frac{\partial u_{2}+u_{h}}{\partial y}-\frac{\partial v_{2}+v_{h}}{\partial x}\right)\right] \\
& =-\mathbf{V} \cdot \nabla\left[\frac{1}{2}\left(\frac{\partial v_{1}}{\partial x}\right)^{2}+\frac{1}{2}\left(\frac{\partial u_{1}}{\partial y}\right)^{2}\right]+\mathbf{V} \cdot \nabla\left(\frac{\partial v_{1}}{\partial x} \frac{\partial u_{1}}{\partial y}\right)
\end{aligned}
$$


With the rotational wind, divergent wind and environmental wind are involved in all of the above three equations (Eqs. (11)-(13)) to substitute the total wind, the opposite-sign terms can be separated from other terms and Eqs. (11)-(13) can be rewritten as

$\frac{\partial \varepsilon}{\partial t}=F_{\zeta R}+F_{\zeta E}+F_{\zeta D}$

$\frac{\partial \delta}{\partial t}=F_{\mathrm{DR}}+F_{\mathrm{DE}}-F_{\zeta \mathrm{D}}$,

$\frac{\partial \sigma}{\partial t}=F_{\mathrm{ER}}-F_{\zeta \mathrm{E}}-F_{\mathrm{DE}}$.

From the above equations,

$$
\begin{aligned}
F_{\zeta E} & =\mathbf{V} \cdot \nabla\left(\frac{\partial v_{1}}{\partial x} \frac{\partial u_{1}}{\partial y}\right)+\frac{\partial v_{1}}{\partial x} \frac{\partial u_{1}}{\partial y} D \\
& +\frac{\partial v_{1}}{\partial x} \frac{\partial \omega}{\partial y} \frac{\partial u}{\partial p}+\frac{\partial u_{1}}{\partial y} \frac{\partial \omega}{\partial x} \frac{\partial v}{\partial p},
\end{aligned}
$$

is the opposite-sign term between the SV equation and the SE equation, and is called the SV-SE interaction term.

$F_{\zeta D}=-f \zeta D+\frac{\partial v_{1}}{\partial x} \frac{\partial u_{1}}{\partial y} D$

is the opposite-sign term between the SV equation and the $\mathrm{SD}$ equation, which is called the SV-SD interaction term.

$$
\begin{aligned}
F_{\mathrm{DE}}= & -\mathbf{V} \cdot \nabla\left(\frac{\partial u_{2}}{\partial x} \frac{\partial v_{2}}{\partial y}\right)-\frac{\partial u_{2}}{\partial x} \frac{\partial v_{2}}{\partial y} D \\
& -\left(\frac{\partial u_{2}}{\partial x} \frac{\partial \omega}{\partial y} \frac{\partial v}{\partial p}+\frac{\partial v_{2}}{\partial y} \frac{\partial \omega}{\partial x} \frac{\partial u}{\partial p}\right)-g\left(\frac{\partial v_{2}}{\partial y} \frac{\partial^{2} z}{\partial x^{2}}+\frac{\partial u_{2}}{\partial x} \frac{\partial^{2} z}{\partial y^{2}}\right)
\end{aligned}
$$

is the opposite-sign term between the SD equation and the SE equation, which is called the SD-SE interaction term.

Apart from the opposite-sign terms, there are also other terms in Eqs. (19)-(21), which are defined as the residual terms of the corresponding equation:

$$
\begin{aligned}
F_{\zeta R}= & -\frac{1}{2} \mathbf{V} \cdot \nabla\left(\left(\frac{\partial v_{1}}{\partial x}\right)^{2}+\left(\frac{\partial u_{1}}{\partial y}\right)^{2}\right)-\left[\left(\frac{\partial v_{1}}{\partial x}\right)^{2}+\left(\frac{\partial u_{1}}{\partial y}\right)^{2}\right] D \\
& -\left(\frac{\partial v_{1}}{\partial x} \frac{\partial \omega}{\partial x} \frac{\partial v}{\partial p}+\frac{\partial u_{1}}{\partial y} \frac{\partial \omega}{\partial y} \frac{\partial u}{\partial p}\right)
\end{aligned}
$$

is the $\mathrm{SV}$ residual term,

$$
\begin{aligned}
F_{\mathrm{DR}} & =-\frac{1}{2} \mathbf{V} \cdot \nabla\left(\left(\frac{\partial u_{2}}{\partial x}\right)^{2}+\left(\frac{\partial v_{2}}{\partial y}\right)^{2}\right) \\
& -\left(\frac{\partial u_{2}}{\partial x}\right)^{3}-D\left(\frac{\partial\left(u_{1}+u_{h}\right)}{\partial x}\right)^{2}-2 D \frac{\partial u_{2}}{\partial x} \frac{\partial\left(u_{1}+u_{h}\right)}{\partial x} \\
& -\left(\frac{\partial v_{2}}{\partial y}\right)^{3}-D\left(\frac{\partial\left(v_{1}+v_{h}\right)}{\partial y}\right)^{2}-2 D \frac{\partial v_{2}}{\partial y} \frac{\partial\left(v_{1}+v_{h}\right)}{\partial y} \\
& -\frac{\partial v_{1}}{\partial x} \frac{\partial u_{1}}{\partial y} D-2 \frac{\partial v_{1}}{\partial x} \frac{\partial\left(u_{2}+u_{h}\right)}{\partial y} D-2 \frac{\partial\left(v_{2}+v_{h}\right)}{\partial x} \frac{\partial u}{\partial y} D \\
& -\left(\frac{\partial u_{2}}{\partial x} \frac{\partial \omega}{\partial x} \frac{\partial u}{\partial p}+\frac{\partial v_{2}}{\partial y} \frac{\partial \omega}{\partial y} \frac{\partial v}{\partial p}\right)-g\left(\frac{\partial u_{2}}{\partial x} \frac{\partial^{2} z}{\partial x^{2}}+\frac{\partial v_{2}}{\partial y} \frac{\partial^{2} z}{\partial y^{2}}\right)
\end{aligned}
$$

$$
\begin{aligned}
F_{\mathrm{ER}}= & -\mathbf{V} \cdot \nabla\left[\frac{1}{2}\left(\frac{\partial v}{\partial x}\right)^{2}+\frac{1}{2}\left(\frac{\partial u}{\partial y}\right)^{2}+\frac{1}{2}\left(\frac{\partial u}{\partial x}\right)^{2}+\frac{1}{2}\left(\frac{\partial v}{\partial y}\right)^{2}\right]-\mathbf{V} \cdot \nabla\left(\frac{\partial v_{1}}{\partial x} \frac{\partial\left(u_{2}+u_{h}\right)}{\partial y}\right)-\mathbf{V} \cdot \nabla\left(\frac{\partial\left(v_{2}+v_{h}\right)}{\partial x} \frac{\partial u}{\partial y}\right) \\
& +\mathbf{V} \cdot \nabla\left(\frac{\partial\left(u_{1}+u_{h}\right)}{\partial x} \frac{\partial v}{\partial y}\right)+\mathbf{V} \cdot \nabla\left(\frac{\partial u_{2}}{\partial x} \frac{\partial\left(v_{1}+v_{h}\right)}{\partial y}\right)-\left[\left(\frac{\partial v}{\partial x}\right)^{2}+\left(\frac{\partial u}{\partial y}\right)^{2}\right] D-\frac{\partial v_{1}}{\partial x} \frac{\partial u_{1}}{\partial y} D \\
& -2 \frac{\partial v_{1}}{\partial x} \frac{\partial\left(u_{2}+u_{h}\right)}{\partial y} D-2 \frac{\partial\left(v_{2}+v_{h}\right)}{\partial x} \frac{\partial u}{\partial y} D-\left(\frac{\partial\left(u_{1}+u_{h}\right)}{\partial x}-\frac{\partial\left(v_{1}+v_{h}\right)}{\partial y}\right)^{2} D \\
& -2\left(\frac{\partial u_{2}}{\partial x}-\frac{\partial v_{2}}{\partial y}\right)\left[\frac{\partial\left(u_{1}+u_{h}\right)}{\partial x}-\frac{\partial\left(v_{1}+v_{h}\right)}{\partial y}\right] D-\left(\frac{\partial u_{2}}{\partial x}\right)^{3}-\left(\frac{\partial v_{2}}{\partial y}\right)^{3}-\left(\frac{\partial u_{1}}{\partial y} \frac{\partial \omega}{\partial y} \frac{\partial u}{\partial p}+\frac{\partial v_{1}}{\partial x} \frac{\partial \omega}{\partial x} \frac{\partial v}{\partial p}\right) \\
& -\left(\frac{\partial\left(v_{2}+v_{h}\right)}{\partial x}+\frac{\partial\left(u_{2}+u_{h}\right)}{\partial y}\right)\left(\frac{\partial \omega}{\partial y} \frac{\partial u}{\partial p}+\frac{\partial \omega}{\partial x} \frac{\partial v}{\partial p}\right)-\left(\frac{\partial\left(u_{1}+u_{h}\right)}{\partial x}-\frac{\partial\left(v_{1}+v_{h}\right)}{\partial y}\right)\left(\frac{\partial \omega}{\partial x} \frac{\partial u}{\partial p}-\frac{\partial \omega}{\partial y} \frac{\partial v}{\partial p}\right) \\
- & \left(\frac{\partial u_{2}}{\partial x} \frac{\partial \omega}{\partial x} \frac{\partial u}{\partial p}+\frac{\partial v_{2}}{\partial y} \frac{\partial \omega}{\partial y} \frac{\partial v}{\partial p}\right)-2 E_{\mathrm{sh}} g \frac{\partial^{2} z}{\partial x \partial y}-g\left(\frac{\partial u}{\partial x}-\frac{\partial\left(v_{1}+v_{h}\right)}{\partial y}\right) \frac{\partial^{2} z}{\partial x^{2}}+g\left(\frac{\partial u_{1}+u_{h}}{\partial x}-\frac{\partial v}{\partial y}\right) \frac{\partial^{2} z}{\partial y^{2}}
\end{aligned}
$$


is the SE residual term.

In the above derivations, the opposite-sign terms of the SV equation, SD equation and SE equation are all identified, which gives exact expressions of the interactions between vorticity, divergence and deformation. Their changes can be summarized by

$\left\{\begin{array}{ll}F_{\zeta E}>0, & \text { squared deformation } \rightarrow \text { squared vorticity } \\ F_{\zeta E}<0, & \text { squared vorticity } \rightarrow \text { squared deformation }\end{array}\right.$,

$\left\{\begin{array}{l}F_{\zeta D}>0, \quad \text { squared divergence } \rightarrow \text { squared vorticity } \\ F_{\zeta D}<0, \quad \text { squared vorticity } \rightarrow \text { squared divergence }\end{array}\right.$,

$\begin{cases}F_{\mathrm{DE}}>0, & \text { squared deformation } \rightarrow \text { squared divergence } \\ F_{\mathrm{DE}}<0, & \text { squared divergence } \rightarrow \text { squared deformation }\end{cases}$

In Eq. (28), when $F_{\zeta E}>0$, SE will decrease and SV increases. SE is transitioned to SV. In reverse, if $F_{\zeta E}<0$, $\mathrm{SV}$ is transitioned to SE. Therefore, $F_{\zeta E}$ is denoted as the SV-SD interaction term. The same principle applies to Eqs. (29) and (30), which, respectively, give the SV-SD interaction term and the SD-SE interaction term. These terms show that the interaction not only exists between vorticity and divergence, but also between vorticity/divergence and deformation. They are easily calculated and permit a quantitative discussion of the corresponding interactions. In addition, by studying how these interaction terms drive the development of SV, SD and SE, the effects of the interactions among the vorticity, divergence and deformation on the evolution of weather systems can then be studied.

Before the application of the above framework, two problems are clarified here: the separability of SV (or SD) and SE, and the physical meanings of the interaction of SV (or SD) and SE.

\subsection{The separability of SV/SD and SE}

With the aid of the Helmholtz theorem, the total wind is decomposed into the rotational wind and the divergent wind, and can be reconstructed by integrations of only the vorticity and divergence (Chen and Kuo 1992b; Zhou and Cao 2010). Krishnamurti and Ramanathan (1982) derived kinetic energy equations for the two parts of the total wind and identified terms with opposite signs between the two equations, indicating the interaction of the rotational and divergent winds by exchanging kinetic energy. Similar work was also carried out by Ding and Liu (1985), Fu et al. (2011), and Cao et al. (2013). However, as one may find, the deformational wind is not present in these kinetic energy equations. Deformation of the rotational wind and the divergent wind is still evident. Bishop (1996a, b) and Cao et al. (2014) attempted to partition the total wind into the rotational part, divergent part and an "environmental" part. The "environmental" part was non-rotational and non-divergent, so it could largely be characterized by a deformational flow pattern. Nevertheless, it is obvious that this "environmental" part does not contain all the deformation, and there are still large parts of deformational motion that implicitly exist in the rotational and divergent winds. In this circumstance, the exchange between the rotational/ divergent wind and the deformational wind is inappropriate. However, it is worth elucidated that the research of this study is not from the angle of the motion itself, but the features of the motion. Vorticity, divergence and deformation are different concepts from the rotational motion, the divergent motion and the deformation motion. They are the differentiation properties of the motion. Vorticity describes the overall rotation of the air particle, divergence denotes the expansion or contraction of the air particle area and deformation represents the change of the shape of the air particle. If one wants to roundly understand the features of the air motion which is continuous fluid, the deformation cannot be ignored. Specific illustrations of the physical meanings of vorticity, divergence and deformation is given in the "Appendix", which further evidences that deformation (SE) is not part of the vorticity (SV) or part of the divergence (SD). The discussion of interactions between vorticity/divergence and deformation in this paper is thus valid.

\subsection{Physical meanings of the interactions of SV/SD and SE}

For 2D turbulence, Okubo (1970) and Weiss (1991) derived a kinematic criterion called Okubo-Weiss criterion. According to the criterion, the regions of the nondivergent physical space are separated into "strain-dominated regions" and "vorticity-dominated regions". In the "strain-dominated regions", the deformation exceeds the vorticity $\left(E^{2}>\zeta^{2}\right)$, and the fluid is in a hyperbolic mode of motion that can strongly shears the vorticity. The spatial gradients of the vorticity, therefore, tend to grow exponentially fast, which is related to a transfer of enstrophy (vorticity) to the small-scale motion of the fluid. Conversely, in the "vorticity-dominated regions", the vorticity exceeds the deformation $\left(E^{2}<\zeta^{2}\right)$, and the fluid is in an elliptical mode of motion that advects the vorticity smoothly. The Okubo-Weiss criterion has been used to explain some phenomena in atmosphere such as the trough thinning and filamentation in tropical cyclones (Rozoff et al. 2006; Wang 
2008; Tsai et al. 2010). It indicates that different values of deformation and vorticity could construct different flow patterns (deformation dominated or vorticity dominated) which then endure different weather developments. Therefore, it is important to discriminate the features of the flow patterns, which is actually an easy task just by calculating the $\zeta^{2}$ and $E^{2}$ (or SV and SE), and by comparing the values of these quantities. However, a more significant task is to discriminate which pattern the current flow will evolve to, so as to guide the weather forecast.

By thoroughly examining the derivation of the SV-SE interaction term, it can be found that the SV-SE interaction term can be traced back to the terms with opposite signs in the definitions of SV and SE as shown by the blue terms in Eqs. (3)-(5). Time tendencies of the term $\frac{\partial v}{\partial x} \frac{\partial u}{\partial y}$ [blue terms in Eqs. (3) and (5)] largely explain the interaction between SV and SE. As these blue terms are deduced from a difference of the vorticity square and the shearing deformation square $\left(\frac{1}{2}\left(\mathrm{SE}_{R}-\mathrm{SV}\right)\right)$, it is denoted as the $\mathrm{SV}-\mathrm{SE}_{\mathrm{R}}$ difference. When $\mathrm{SV}$ is transitioned to $\mathrm{SE}\left(F_{\zeta E}<0\right)$, time tendency of the SV-SE $E_{\mathrm{R}}$ difference has $\frac{\partial}{\partial t}\left[\frac{1}{2}\left(\mathrm{SE}_{\mathrm{R}}-\mathrm{SV}\right)\right]>0$, meaning $\frac{\partial}{\partial t} \mathrm{SE}_{\mathrm{R}}>\frac{\partial}{\partial t} \mathrm{SV}$. It represents that the increase of $\mathrm{SE}_{\mathrm{R}}$ is larger than the increase of $\mathrm{SV}$, or the decrease of $\mathrm{SE}_{\mathrm{R}}$ is smaller than the decrease of SV. Without considering the stretching deformation and the divergence, the flow will evolve toward a (shearing) deformation-dominated pattern. Conversely, when $\mathrm{SE}_{\mathrm{R}}$ is transitioned to $\mathrm{SV}\left(F_{\zeta E}>0\right)$, $\frac{\partial}{\partial t} \mathrm{SE}_{\mathrm{R}}<\frac{\partial}{\partial t} \mathrm{SV}$ can be obtained through the SV-SE $\mathrm{R}_{\mathrm{R}}$ difference term's time tendency, meaning the flow will evolve toward a vorticity-dominated pattern.

For the SD-SE interactions, the principle is similar. The SD-SE interaction term is mostly explained by the time tendencies of the red terms in Eqs. (4)-(5), which is deduced from the difference of $\mathrm{SD}$ and $\mathrm{SE}_{\mathrm{S}}$ (called $\mathrm{SD}-\mathrm{SE}_{\mathrm{S}}$ difference). When $\mathrm{SD}$ is transitioned to $\mathrm{SE}\left(F_{\mathrm{DE}}<0\right)$, time tendency of the SD-SE $E_{S}$ difference term has $\frac{\partial}{\partial t}\left[\frac{1}{2}\left(\mathrm{SD}-\mathrm{SE}_{\mathrm{S}}\right)\right]<0$, meaning the increase of $\mathrm{SD}$ is smaller than the increase of $\mathrm{SE}_{\mathrm{S}}\left(\frac{\partial}{\partial t} \mathrm{SE}_{\mathrm{S}}>\frac{\partial}{\partial t} \mathrm{SD}>0\right)$ or the decrease of $\mathrm{SD}$ is larger than the decrease of $\mathrm{SE}$ $\left(\frac{\partial}{\partial t} \mathrm{SD}<\frac{\partial}{\partial t} \mathrm{SE}_{\mathrm{s}}<0\right)$.If we tentatively do not consider the shearing deformation and the vorticity, the flow tends to evolve toward the (stretching) deformation-dominated pattern. On the contrary, when SE is transitioned to SD, the flow will then evolve toward the divergence-dominated pattern.

The above analysis present that the SV-SE interaction term and the SD-SE interaction to some extent represent an evolution potential of the current flow which is denoted by the time tendencies of the SV-SE $E_{R}$ difference and the $\mathrm{SD}-\mathrm{SE}_{\mathrm{S}}$ difference, respectively. The factors that can induce the change of $\mathrm{SV}-\mathrm{SE}_{\mathrm{R}}$ difference is mostly contained in the
SV-SE interaction term, including the SV-SE difference advection, the divergence of the SV-SE difference and a tilting effect. The factors controlling the change of SD-SE $\mathrm{S}_{\mathrm{S}}$ difference is mostly contained in the SD-SE interaction term, including the $\mathrm{SD}-\mathrm{SE}_{\mathrm{S}}$ difference advection, the divergence of the $\mathrm{SD}-\mathrm{SE}_{\mathrm{S}}$ difference, the tilting effect and the quadratic differential term of geopotential height.

\section{Case study}

In this section, the effects of the interactions among vorticity, divergence and deformation on the evolution of a precipitating vortex are studied. The vortex, which occurs during 16-17 August 2009, is characterized by a typical meso- $\alpha$ scale of approximately $400-600 \mathrm{~km}$ and long-term maintenance. It induced a severe precipitation during the passage of Shandong province, with the 24-h precipitation up to $300 \mathrm{~mm}$ (Yang et al. 2013). The data we used for the calculation and analysis come from the National Centers for Environmental Prediction (NCEP) Global Forecasting System (GFS) analysis dataset, with a horizontal grid spacing of $0.5^{\circ}$ and a temporal frequency of $6 \mathrm{~h}$.

\subsection{Case overview}

Figure 1 shows the horizontal distributions of the wind streams, SV and precipitation at $800 \mathrm{hPa}$ to illustrate the evolution of the vortex. In Fig. 1, we mainly use SV to denote the intensity of the vortex, but the minimum geopotential height is also considered, although not shown. According to the stream lines and SV in Fig. 1, the studied meso- $\alpha$ vortex (enclosed by the black box, Fig. 1b) initiates at about 0000 UTC 17 August 2009 at $\left(35.5^{\circ} \mathrm{N}, 113.5^{\circ} \mathrm{E}\right)$. From the flow pattern before initiation in Fig. 1a, the vortex seems to have developed from a cyclonic veer of a southwesterly wind. In Fig. 1a, we can see that at 1800 UTC 16 August an evident confluence and southerly-southeasterly cyclonic shear exist near to where the vortex is generated later (black triangle). Figure 2 shows the basic large-scale environment in which the vortex developed, with the vortex area enclosed by the dashed black box. As shown in Fig. 2a, at 0000 UTC 17 August, when the vortex initiates, the most evident feature of the 500-hPa pattern is a westward and northward extension of the subtropical high (5880 gpm contour), the west border of which reaches the southeast border of China. A shallow low trough exists on the northwest of the subtropical high. At 0000 UTC 17 August, the vortex (enclosed by the dashed black box) is located in front of the $500-\mathrm{hPa}$ low trough, where ascending flow tends to develop. At $800 \mathrm{hPa}$, the vortex is under the influence of a strong wind band (colorshaded area), which can be viewed as a conveyor belt of kinetic energy and moisture (stream lines in Fig. 1a) into the 
(a) 18 UTC, August 16

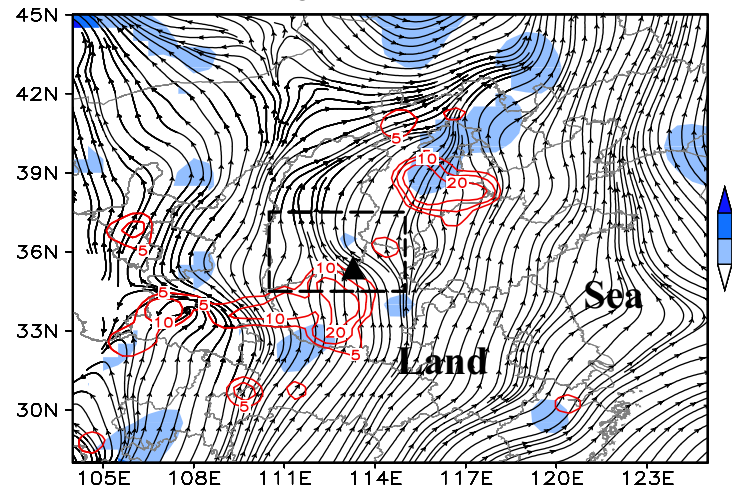

(b) 00 UTC, August 17

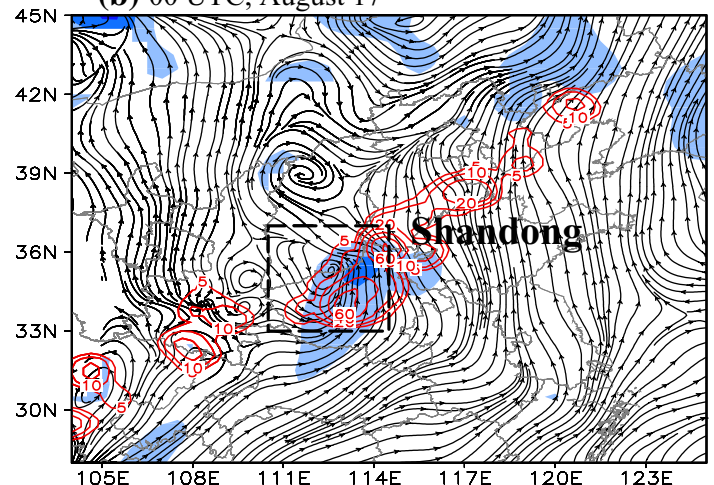

(c) 06 UTC, August 17

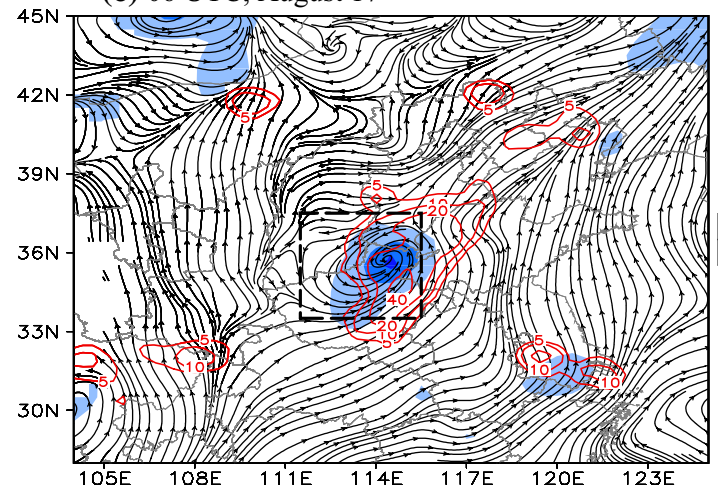

(d) 12 UTC, August 17

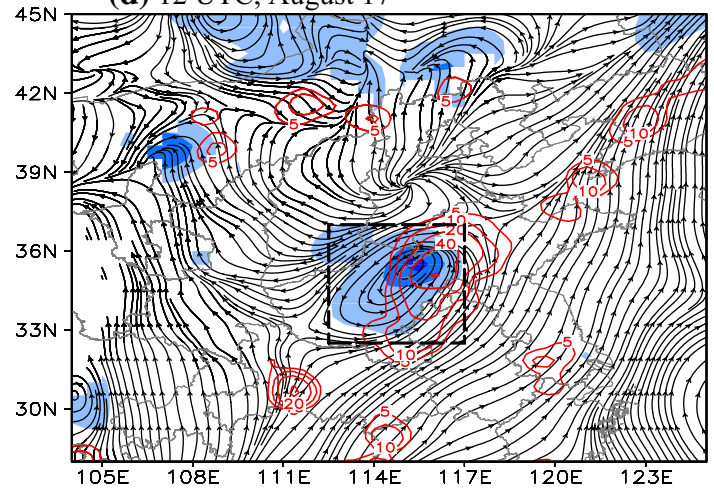

Fig. 1 SV (color-shaded area, units: $10^{-9} \mathrm{~s}^{-2}$ ) and stream lines at $800 \mathrm{hPa}$ from 1800 UTC 16 August to 1200 UTC 18 August 2009 every $6 \mathrm{~h}$. The black boxes enclose the whole vortex area. The red (e) 18 UTC, August 17

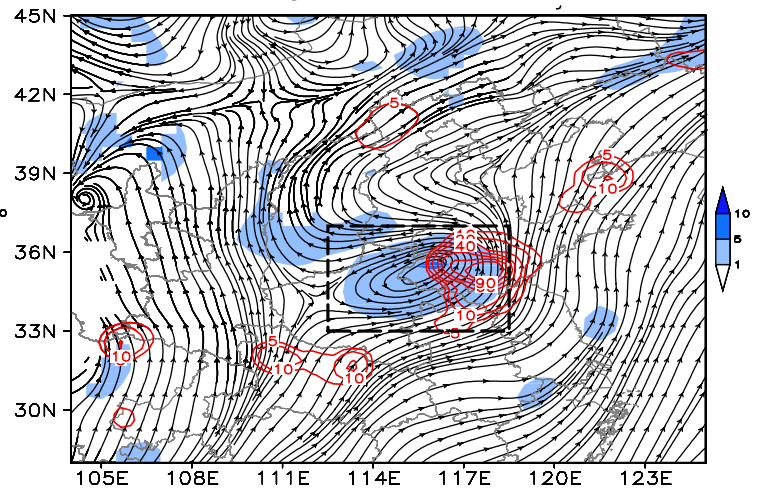

(f) 00 UTC, August 18

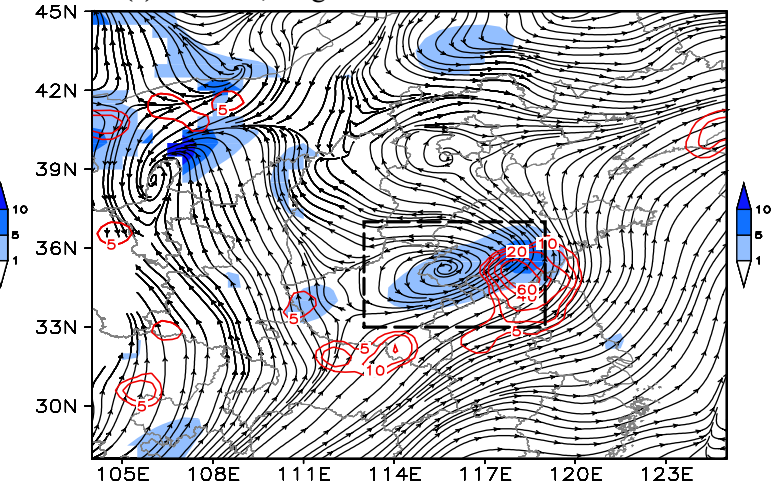

(g) 06 UTC, August 18

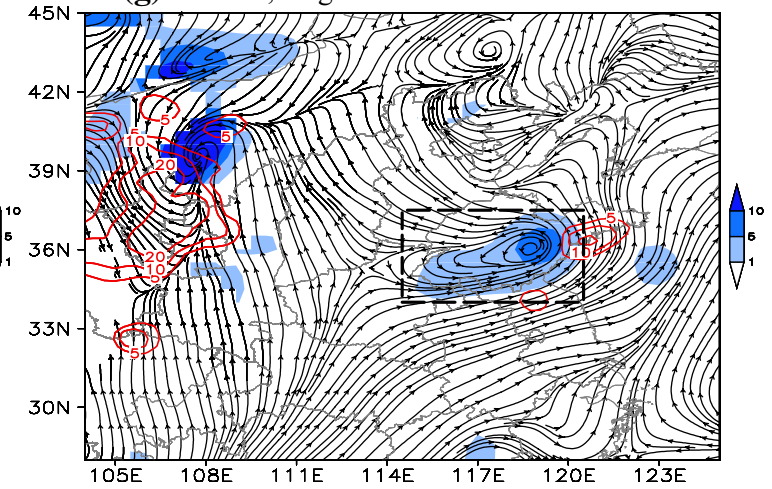

(h) 12 UTC, August 18

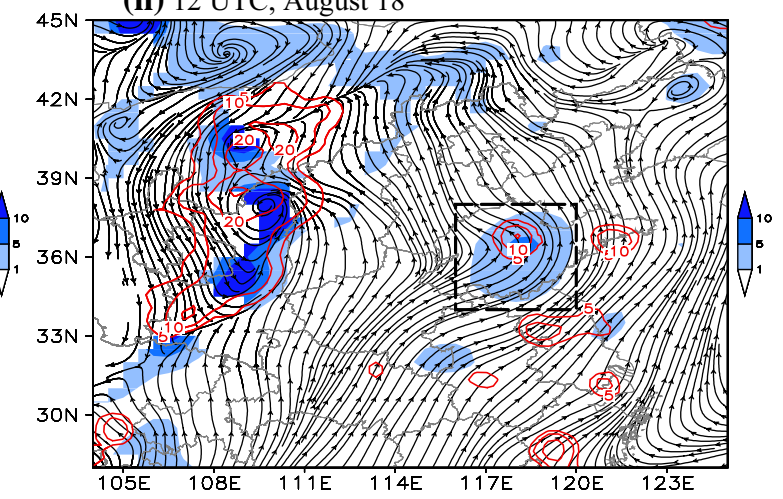

lines are the 6-h accumulated rainfall amount $(\mathrm{mm})$. The triangle symbol in Fig. 1 indicates the formation location of the vortex 

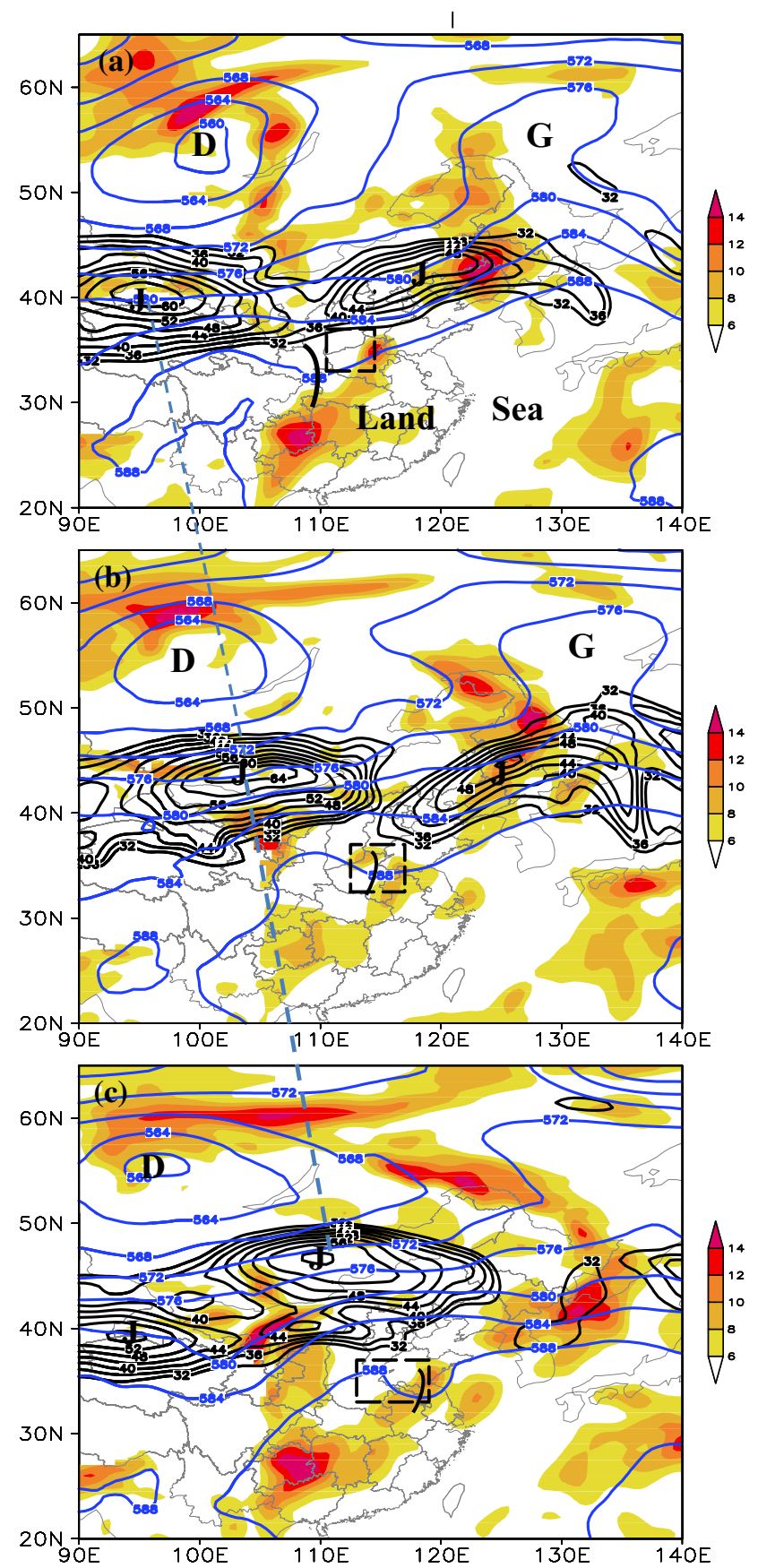

Fig. 2 800-hPa wind speed (color-shaded areas, units: $\mathrm{m} \mathrm{s}^{-1}$ ), geopotential height at $500 \mathrm{hPa}$ (blue solid lines, units: $10 \mathrm{gpm}$ ), and 200$\mathrm{hPa}$ wind speed (black solid lines, units: $\mathrm{m} \mathrm{s}^{-1}$ ) at a 0000 UTC 17 August, b 1200 UTC 17 August, and c 0000 UTC 18 August 2009. "G" denotes the high ridge, "D" denotes the extratropical cyclone and "J" is the high-level jet. The black dashed boxes represent the vortex area. The black arc line is the $500-\mathrm{hPa}$ low trough

vortex area and also the precipitation area, although it does not reach the intensity of a low-level jet stream $(>12 \mathrm{~m} / \mathrm{s})$. At $200 \mathrm{hPa}$, two high-level jet streams exist at about $40^{\circ} \mathrm{N}$ over China. The vortex is located on the right-hand side of the entrance of the eastern jet, where the divergence of the flow is usually remarkable. The pumping effect due to the high-level divergence, the ascending motion due to the mid-level low trough and the moisture transport due to the strong wind band are features that are quite favorable for the development of low-level vortices and precipitation.

After initiation, the vortex moves eastward, intensifies, and induces heavy precipitation along its path (Fig. 1a-d). As shown in Fig. 1d, at 1200 UTC 17 August, the vortex reaches the western border of Shandong Province $\left(115^{\circ} \mathrm{E}\right)$, with the maximum SV collocated with the center of the cyclonic circulation. The precipitation area, which has an evident southwest-northeast orientation, is mainly located on the east and southeast sides of the vortex. Compared with 0000 UTC 17 August, both the upper- and lower-level systems have significant eastward displacements correspondingly. As shown in Fig. 2b, the two jet cores at $200 \mathrm{hPa}$ move about $1000 \mathrm{~km}\left(10^{\circ}\right.$ of latitude $)$ in $12 \mathrm{~h}$, with the vortex (enclosed by the black box) located between the two jet streams. The 500-hPa subtropical high withdraws eastward, with the low trough also moving eastward through the lowlevel vortex area. Heavy precipitation mainly occurs in front of the low trough where there are upper-level divergence due to the high-level jet, low-level convergence due to the vortex and strong low-level wind (Fig. 2b). The low trough may have played an important role in the upward development of the vortex and the long lifespan of the vortex.

After its intensification, the vortex stagnates over the west of Shandong, which then causes extreme precipitation (Fig. 1e-f). After that, the vortex begins to dissipate (Fig. 1g, h). As shown in Fig. 2c, at $200 \mathrm{hPa}$, due to the acceleration of the eastern jet, the vortex (enclosed by the black box) is exposed in the exit area of the western jet, which is characterized by high-level convergence. The $500-\mathrm{hPa}$ low trough has passed through the vortex area, and the subtropical high withdraws eastward. Due to the high-level convergence and descending flow behind the mid-level low trough, the vortex and associated precipitation begin to dissipate (Fig. 1f-h).

According to the evolution of the SV and the flow pattern in Fig. 1, as well as the evolution of the large-scale weather pattern in Fig. 2, the complete lifespan of the meso- $\alpha$-scale vortex can be decomposed into four stages: the initiation stage, intensification stage, maturation, and dissipation stage (see Table 1). As shown in Table 1, the vortex initiates at $800 \mathrm{hPa}$ in the lower troposphere of the atmosphere. The vertical extension of the vortex (determined by examining the closed streams lines and geopotential height contours in each level) then enlarges with its intensification and contracts with its dissipation. It is important to note that, although 1800 UTC 17 August is the time defined as the maturation stage of the vortex, it does not actually entirely conform to the characteristics of maturation, with the SV 
Table 1 The evolution stage, vertical extent, and horizontal area of the vortex

\begin{tabular}{llll}
\hline Time & Stage & Vertical extension & Horizontal vortex area \\
\hline 1800 UTC 16 August, 2009 & Before initiation & $800 \mathrm{hPa}$ & $\left(34.5^{\circ}-37.5^{\circ} \mathrm{N}, 110.5^{\circ}-115^{\circ} \mathrm{E}\right)$ \\
0000 UTC 17 August, 2009 & Initiation & $850-800 \mathrm{hPa}$ & $\left(33^{\circ}-37^{\circ} \mathrm{N}, 110.5^{\circ}-114.5^{\circ} \mathrm{E}\right)$ \\
0600 UTC 17 August, 2009 & Intensification & $975-700 \mathrm{hPa}$ & $\left(33.5^{\circ}-37.5^{\circ} \mathrm{N}, 111.5^{\circ}-115.5^{\circ} \mathrm{E}\right)$ \\
$1200 \mathrm{UTC} 17$ August, 2009 & Intensification & $975-600 \mathrm{hPa}$ & $\left(32.5^{\circ}-37^{\circ} \mathrm{N}, 112.5^{\circ}-117^{\circ} \mathrm{E}\right)$ \\
1800 UTC 17 August, 2009 & Maturation & $975-600 \mathrm{hPa}$ & $\left(33^{\circ}-37^{\circ} \mathrm{N}, 112.5^{\circ}-118.5^{\circ} \mathrm{E}\right)$ \\
0000 UTC 18 August, 2009 & Dissipation & $975-650 \mathrm{hPa}$ & $\left(33^{\circ}-37^{\circ} \mathrm{N}, 113^{\circ}-119^{\circ} \mathrm{E}\right)$ \\
0600 UTC 18 August, 2009 & Dissipation & $975-650 \mathrm{hPa}$ & $\left(34^{\circ}-37.5^{\circ} \mathrm{N}, 114.5^{\circ}-120.5^{\circ} \mathrm{E}\right)$ \\
1200 UTC 18 August, 2009 & Dissipation & $975-700 \mathrm{hPa}$ & $\left(34^{\circ}-38^{\circ} \mathrm{N}, 116^{\circ}-120^{\circ} \mathrm{E}\right)$
\end{tabular}

decreasing compared to 1200 UTC. This means that the maturation of the vortex is not so evident, or the maturation of the vortex is not reflected in the current data due to the low temporal resolution $(6 \mathrm{~h})$. Therefore, in the following analysis, only three stages of the vortex are analyzed: the initiation stage, developing stage and dissipation stage.

\subsection{Analysis of the SV equation}

The SV equation is used to diagnose the factors influencing the evolution of the vortex from the angle of the interactions of vorticity, divergence and deformation. Figure 3 illustrates the horizontal distributions of the total forcing $\left(F_{\zeta D}+F_{\zeta E}+F_{\zeta R}\right)$ of the SV equation and its three components: the SV-SD interaction term $\left(F_{\zeta D}\right)$, the SV-SE interaction term $\left(F_{\zeta E}\right)$, and the residual term of the SV equation $\left(F_{\zeta R}\right)$ at $800 \mathrm{hPa}$ in the initiation stage (1800 UTC 16 August), intensification stage (0600 UTC 17 August), and dissipation stage (0600 UTC 18 August). Before the detailed analysis, it is noted that here we use the total forcing instead of time derivative of SV to denote the vortex evolution. This is mainly because in our study, 6-h NCEP reanalysis data are used. In the initiation stage and the dissipation stage of the vortex which might be slow-manifold processes, the left side of the SV equation can balance the right side well. However, in the intensification stage, the right side of the SV equation is much larger than the left side. This means that the rapid development of SV during a short time may not be reflected by the time derivative calculated from the 6-h NCEP reanalysis data. Therefore, we use the total forcing to reflect this rapid development process.

As is shown, at 1800 UTC 16 August, when the vortex develops at approximately $\left(35.5^{\circ} \mathrm{N}, 113.5^{\circ} \mathrm{E}\right)$, marked by the black dot in Fig. 1a, most of the vortex area has positive values of total SV forcing $\left(F_{\zeta D}+F_{\zeta E}+F_{\zeta R}\right.$, red lines), with two maximum centers of $1.8 \times 10^{-13} \mathrm{~s}^{-2}$ and $1.2 \times 10^{-13} \mathrm{~s}^{-2}$, respectively. These positive values indicate increases in $\mathrm{SV}$ in the corresponding regions, which then induces the formation of the vortex (Fig. 2a, b).
Figure $3 b-d$ presents the distributions of the three components of the total SV forcing. From Fig. 3b-d, it can be seen that all three components make positive contributions to the formation of the vortex. The SV-SD interaction term in Fig. $3 \mathrm{~b}$ mainly shows positive values in the vortex area and has a similar distribution pattern to the total forcing (Fig. 3a), with two maximum centers of $1.2 \times 10^{-13} \mathrm{~s}^{-2}$ and $0.9 \times 10^{-13} \mathrm{~s}^{-2}$, respectively. According to Eq. (29), positive values of SV-SD interaction term represent a transition of SD-SV. In this process, the SD is consumed, while the SV tends to increase, which is favorable for the initiation of a vortex. The SV-SE interaction term in Fig. 3c shows a very different distribution pattern from the SV-SD interaction term. In Fig. 3c, the zero contour of the SV-SE interaction term passes through the vortex region. Southeast of the zero contour is the positive-value area, with a maximum of $0.3 \times 10^{-13} \mathrm{~s}^{-2}$. In this area, the SE transforms to SV according to Eq. (28), making a positive contribution to the vortex formation (black dot). The residual term of the SV equation also shows a positive value in the vortex initiation area (black dot), meaning SV is generated to drive the initiation of the vortex. According to the above analysis, three effects contribute to the initiation of the meso- $\alpha$-scale vortex: the transition of divergence to vorticity, deformation to vorticity, and the generation of SV by the residual term of the SV equation. Among these terms, the transition of SD to SV plays the most important role in the initiation of the vortex. The transition of SE to SV is second most important, while the generation of $\mathrm{SV}$ is least important. The first two terms describe the interactions of vorticity, divergence and deformation, which are demonstrated to have a significant role in the formation process of this meso- $\alpha$-scale vortex.

After initiation, the vortex undergoes a rapid development stage. As shown in Fig. 3e, positive values of total SV forcing at 0600 UTC 17 August increases remarkably compared to during the formation stage, with the maximum value being $1.3 \times 10^{-12} \mathrm{~s}^{-2}$. In Fig. 3e, it is apparent that the vortex (black box) is separated into two parts, approximately by the zero line of total SV forcing. East of the zero line, occupied by most parts of the vortex, is a large positivevalue area of total SV forcing, and in this area, the vortex 

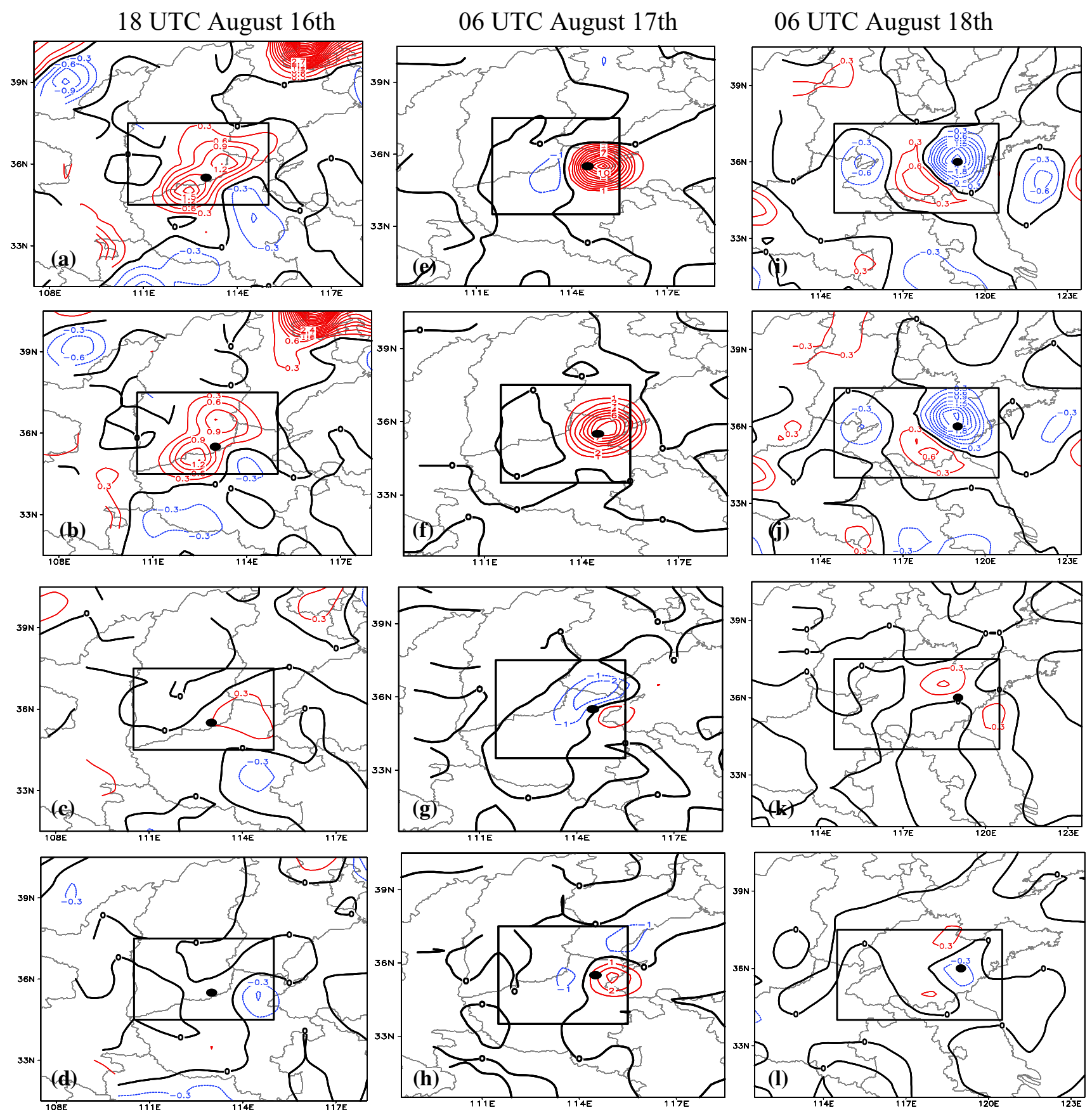

Fig. 3 a, e, i The total forcing (sum of the terms on the right-hand side of the vorticity equation), $\mathbf{b}, \mathbf{f}, \mathbf{j} \mathrm{SV}-\mathrm{SD}$ interaction term, $\mathbf{c}, \mathbf{g}$, $\mathbf{k}$ SV-SE interaction term, and $\mathbf{d}, \mathbf{h}, \mathbf{l}$ residual term of the SV equation at $800 \mathrm{hPa}$ at 1800 UTC 16 August, 0600 UTC 17 August, and

tends to develop rapidly through the increase of SV. West of the zero line is a negative-value area where the SV tends to decrease and the vortex weakens. Due to the spatial distribution pattern of the total SV forcing, i.e. the negative-value area following the positive-value area, the vortex tends to move eastward, which can also be verified by Fig. 2c. Similar to the initiation stage, to identify the essential reason
0600 UTC 18 August 2009. The red lines indicate positive values, the blue lines indicate negative lines, and the black lines are the zero lines. The black boxes are the vortex area. The black dots indicate the approximate locations of the vortex center. Units: $10^{-13} \mathrm{~s}^{-2}$

for the rapid development and eastward motion of the vortex, Fig. 3f-h illustrates the three components of the total $\mathrm{SV}$ forcing. From Fig. 3f-h, we can see that the leading factor that drives the development of the vortex is still the transition of SD to SV. In Fig. 3f, the SV-SD interaction term shows strong positive values in the vortex influence area of the vortex, with the maxima having increased by 
about $7 \times 10^{-13} \mathrm{~s}^{-2}$ compared with the initiation stage. The SV-SE interaction term and residual term also show positive values in the vortex area, but these are not as intensive as the SV-SD interaction term. In Fig. 3g, the zero line of the SV-SE term passes through the vortex centers. East of the zero lines where the SV-SE term shows positive values, the SE transforms to SV to drive the development of the vortex; while west of the zero line, the negative values of the SV-SE term causes a transition of SV-SE to reduce the intensification of the vortex. The residual term of the SV equation has a similar pattern to the SV-SE term, which also shows positive values east of the vortex and negative values west of the vortex, except the zero line bypassing the vortex center. Through the above analysis, we can see that the pattern of the negative-value area following the positivevalue area also applies to the SV-SE transition term and the residual term of the SV equation. This means that, although these two terms do not have decisive effects on the development of the vortex, they may lead its eastward propagation.

Figure 3i-l illustrates the total SV forcing and its three components at 06 UTC, August 18th, in the dissipation stage of the vortex. At this time, the meso- $\alpha$-scale vortex still holds a complete closed cyclonic circulation in the middle of Shandong province (Fig. 1g). After $6 \mathrm{~h}$ (Fig. 1h), the closed circulation disappears, and an east-west oriented shear line stays. Corresponding to this process, the total forcing of SV presents a strong negative center, which is collocated with the vortex center, northeast of the vortex area. In these negative-value areas, the SV decreases and the vortex dissipates. From Fig. $3 j-1$, the main factor affecting the dissipation of the vortex is due to the SV-SD interaction term, i.e. the transformation of $\mathrm{SV}$ to SD. Another factor that plays a role in the dissipation of the vortex is the residual term of the SV equation, which also shows a negative-value center in the vortex center. However, these negative values are much weaker than those of the SV-SD interaction term. Contrary to the above two terms, the SV-SE interaction term shows a very different effect on the dissipation of the vortex. Similar to during the intensification stage, in Fig. 3k, the zero line of the SV-SE interaction term passes through the vortex center. North of the zero line, the SE transforms to $\mathrm{SV}$ to sustain the vortex. South of the zero line, the SV is consumed and transforms to SE, causing the vortex to dissipate. This pattern of a negative value following a positive value favors the northwestward movement of the vortex.

Through all the above analyses of the SV equation during the complete lifespan of this meso- $\alpha$-scale vortex, one can conclude that the main factor that influenced its evolution was the SV-SD interaction term. In the initiation stage and intensification stage, this term presented strong positive values inside the vortex area, which drove the SD to transform to SV to intensify the rotation. In the dissipation stage, this term became negative in the vortex center where the SV transformed to SD to weaken the rotation. This result is consistent with the relation between vorticity and divergence that has obtained from the divergence term of the vertical vorticity equation (Holton 2004). The effects of the SV-SE interaction term and residual term of the SV equation seem to be much weaker than the SV-SD interaction term. However, both presented a distribution pattern of a negative value following a positive value, which may have led to the vortex moving toward the positive-value area.

\subsection{Analysis of the SV-SD interaction term}

The above analyses of the SV equation show that, through transforming to SV, deformation on the one hand directly drives the development of the vortex, while on the other hand may have influenced its movement. However, whether SV-SE interaction is the only way that deformation influences the development of the vortex still needs further investigation. For this reason, the main mechanism that controls the interaction between $\mathrm{SV}$ and $\mathrm{SD}$, which is the leading factor affecting the evolution of the vortex, is discussed in this section. Consider the SV-SD interaction term in Eqs. (19) and (20) only:

$\frac{\mathrm{d} \varepsilon}{\mathrm{d} t} \sim-f \zeta D+\frac{\partial v_{1}}{\partial x} \frac{\partial u_{1}}{\partial y} D$,

$\frac{\mathrm{d} \delta}{\mathrm{d} t} \sim f \zeta D-\frac{\partial v_{1}}{\partial x} \frac{\partial u_{1}}{\partial y} D$.

Equations (31) and (32) show that the interaction of the SV and SD are related to two terms. The first term is the Coriolis parameter term, which is determined by the configuration of vorticity and divergence. For example, for a cyclonic convergent circulation $(\zeta>0, D<0)$, the Coriolis parameter term is negative $(f \zeta D<0)$. The SD thus transforms to SV to increase the positive cyclonic vorticity. The second term on the right-hand side of Eq. (31) or (32) is the coupling of the divergence and the shear of the rotational wind, $\left(u_{1}, v_{1}\right)$, which is determined by the distribution of the flow. Figure 4 shows the relative distributions of the two subterms of the SV-SD interaction term. According to Figs. 3b, $\mathrm{f}$ and $\mathrm{j}$ and 4 , we can see that the term $f \zeta D$ determines the basic distribution of the SV-SD interaction term. Given that in the vortex area the vertical vorticity $\zeta$ is generally positive, the divergence $D$ thus becomes the decisive factor for the sign of the SV-SD interaction term. In the initiation stage and intensification stage, as shown in Fig. 5a, b, the vortex center (black dot) appears convergent $(D<0)$, which makes $f \zeta D$ negative and the SD to transforms to SV to drive the development of the vortex. In the dissipation stage, the flow becomes divergence in the vortex center (black dot in Fig. $5 \mathrm{c}$ ), and the term $f \zeta D$ is positive, which then makes the 

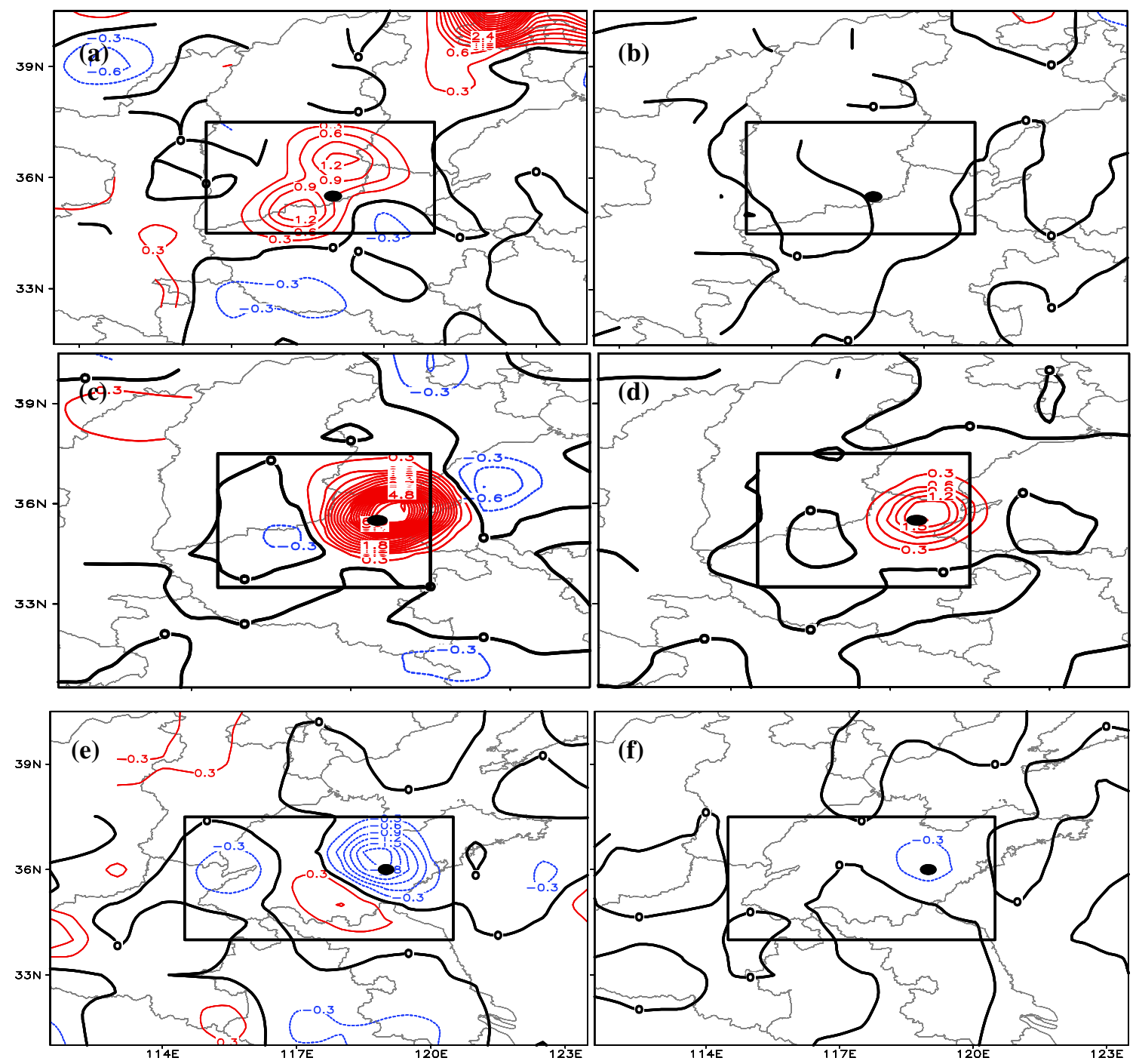

Fig. 4 Horizontal distributions of the two sub-terms of the SV-SD interaction term at $800 \mathrm{hPa}$ at a, b 1800 UTC 16 August, c, d 0600 UTC 17 August, and e, f 0600 UTC 18 August 2009. The first column is the Coriolis parameter-related term, and the second column is the coupling of the divergence and the shear of rotational wind.
The red lines indicate positive values, the blue lines indicate negative lines, and the black lines are the zero lines. The black boxes are the vortex area. The black dots indicate the approximate locations of the center of the vortex. Units: $10^{-13} \mathrm{~s}^{-2}$

August), and dissipation stage (0600 UTC 18 August) of the meso- $\alpha$-scale vortex. As shown in Fig. $6 \mathrm{a}$, in the initiation stage, the total forcing of the SD has positive values in the formation region of the vortex (black dot), meaning SD will increase. Since in this stage the flow in the vortex formation area was convergent, i.e. $D<0$, the increase of SD means an intensification of the convergence of the flow, which then indirectly favors the transition of SD to SV according to Eqs. (31) and (32). Comparing Fig. 6b-d, the positive forcing of the SD is mainly related to the SD-SE interaction term (Fig. 6c) and the residual term of the SD equation (Fig. 6d). As shown, the SV-SD interaction term is mainly negative in the vorticity formation area, which consumes the SD due to the transformation of SD to SV to initiate the (1800 UTC 16 August), intensification stage (0600 UTC 17 

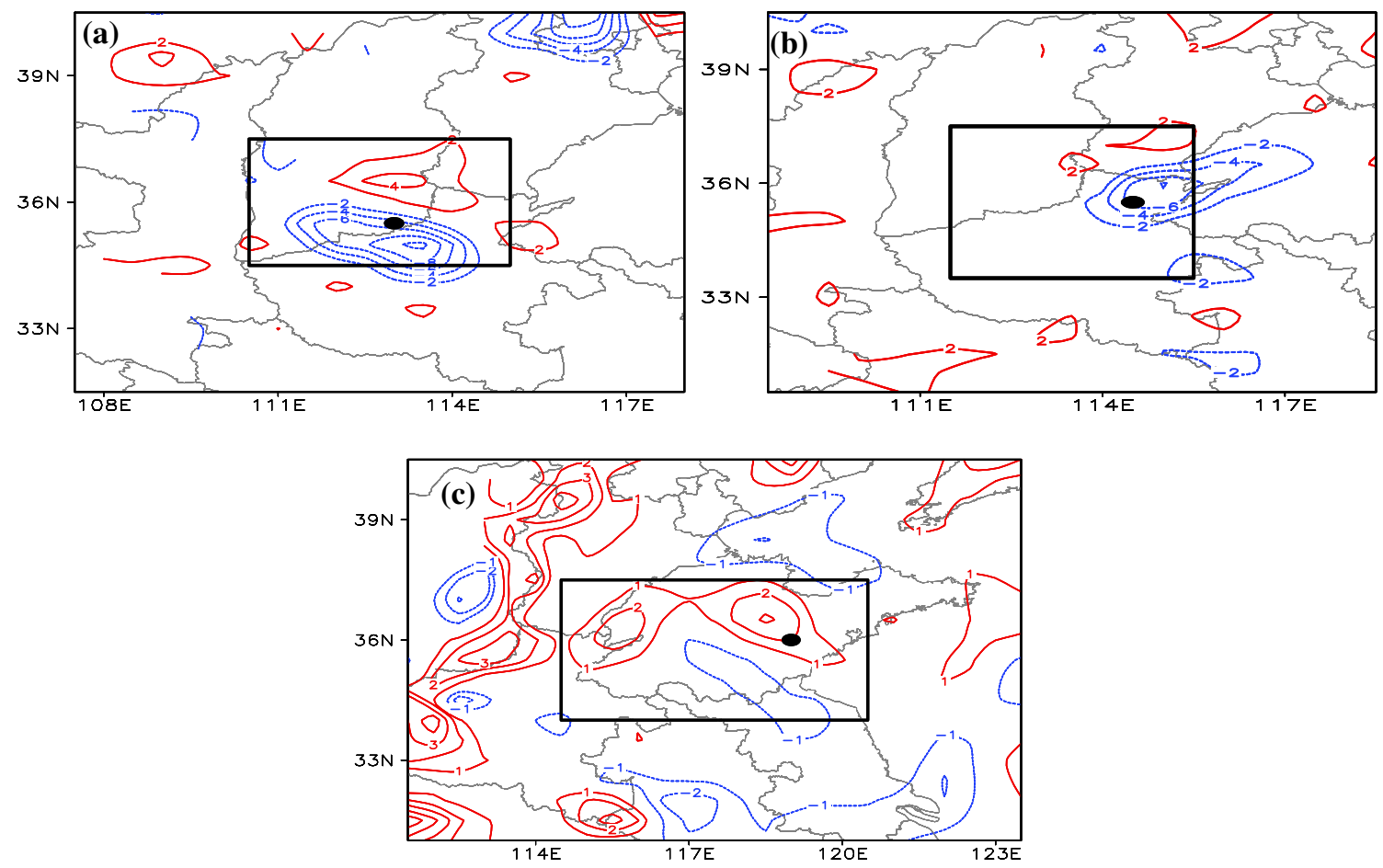

Fig. 5 The divergence at $800 \mathrm{hPa}$ at a 1800 UTC 16 August, b 0600 UTC 17 August, and c 0600 UTC 18 August 2009. The red lines indicate positive values and the blue lines indicate negative lines. The black boxes are the vortex area. The black dots indicate the approximate locations of the center of the vortex. Units: $10^{-5} \mathrm{~s}^{-1}$

the sharp decrease in the residual term of the SD equation (Fig. 6h).

The production of SD by the SD-SE interaction term cannot prevent its large consumption during the rapid intensification of the vortex. Once the SD decreases to zero, i.e. the convergence disappears, the flow may have become divergent. As shown in Fig. 5c, in the dissipation stage, the flow in the vortex area becomes divergent and the SV-SD interaction term changes sign. The SV thus transforms to SD to make the vortex dissipate. As shown in Fig. $6 \mathrm{j}$, the SV-SD interaction term $\left(-F_{\zeta D}\right)$ shows strong positive values in the vortex area (black dot), which drives the transition of the SV to SD to make the divergence increase further. This process accelerates the dissipation of the vortex. The residual term of the SD equation also shows a weak positive value in the vortex center to make the intensity of the divergence increase (black dot) and the vortex dissipate, while the SD-SE interaction term appears negative in the vortex center. These negative values indicate a transition of the SD to SE, which tends to decrease the SD and thus resists an increase in divergence. This in fact indirectly weakens the dissipation of the vortex, according to Eqs. (31) and (32).

From the analyses in this section, we can see that, due to the consumption of SD, the process of the development of 

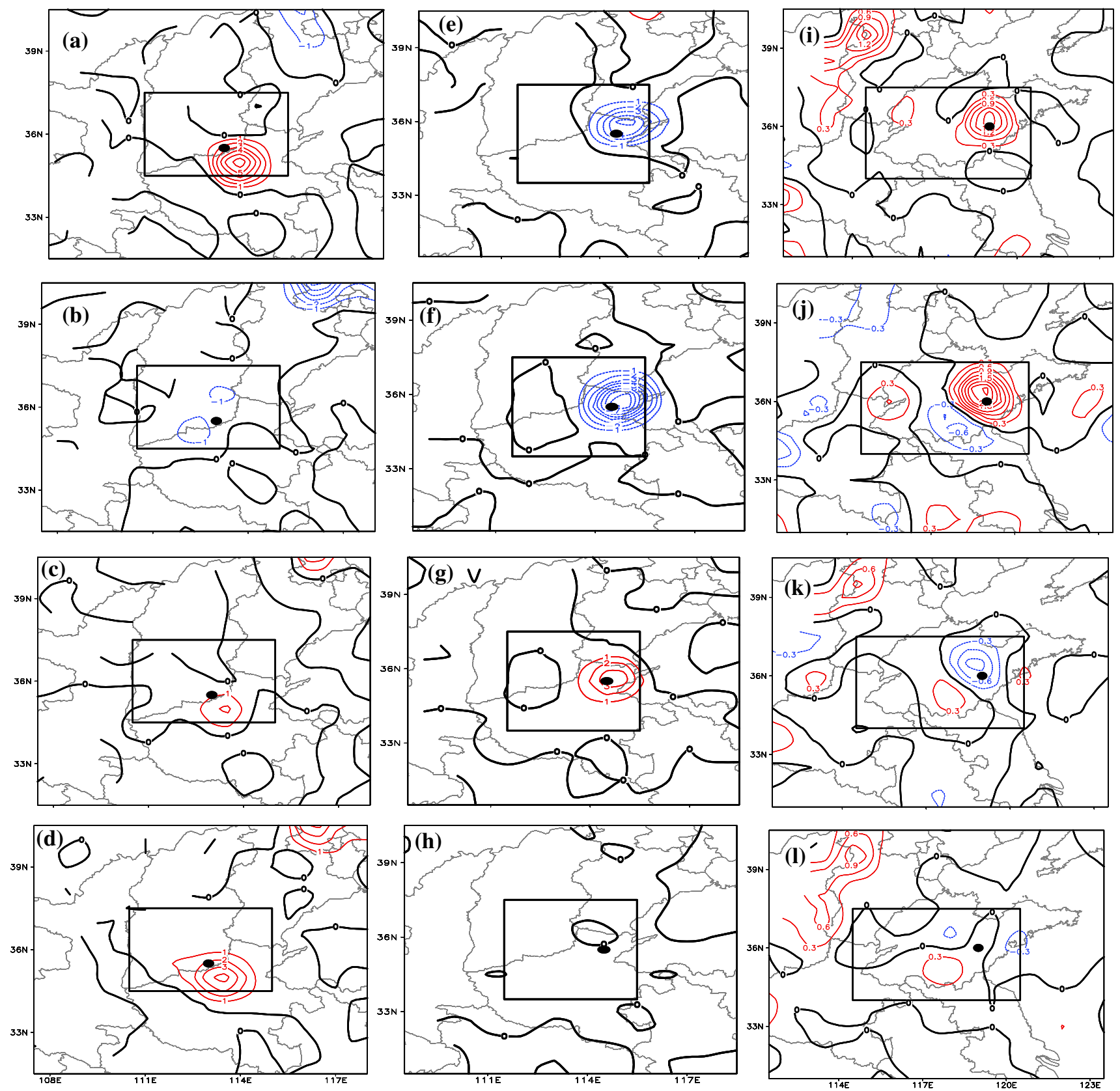

Fig. $6 \mathbf{a}$, e, i The total forcing, b, f, j SV-SD interaction term, $\mathbf{c}, \mathbf{g}$, $\mathbf{k}$ SD-SE, and $\mathbf{d}, \mathbf{h}, \mathbf{l}$ residual term of the SD equation at $800 \mathrm{hPa}$ at 1800 UTC 16 August, 0600 UTC 17 August, and 0600 UTC 18 August 2009. The red lines indicate positive values, the blue lines indicate negative lines, and the black lines are the zero lines. The black boxes are the vortex area. The black dots indicate the approximate locations of the center of the vortex. Units: $10^{-13} \mathrm{~s}^{-2}$ this vortex is actually accompanied by a dissipation mechanism. When the SV of the vortex increases, the SD will be consumed. Once the consumption exceeds the production of $\mathrm{SD}$, the SD will decrease, which may cause the convergent flow to transform to divergent flow, and then initiates the vortex to die out. In this process, deformation seems to play a role in sustaining the rotation of the flow. When the SD is sharply consumed during the development of a vortex, the
SE behaves to transform to SD to fill up its consumption to sustain the convergence of the flow. When the SD increases by the transition from SV during the vortex dissipation stage, the SD transforms to SE to resist an increase of divergence and thus the dissipation of the vortex.

Figure 7 illustrates the budgets of SV, SD and SE around the center of the vortex in each of its stages, which concludes the whole process that has been presented 

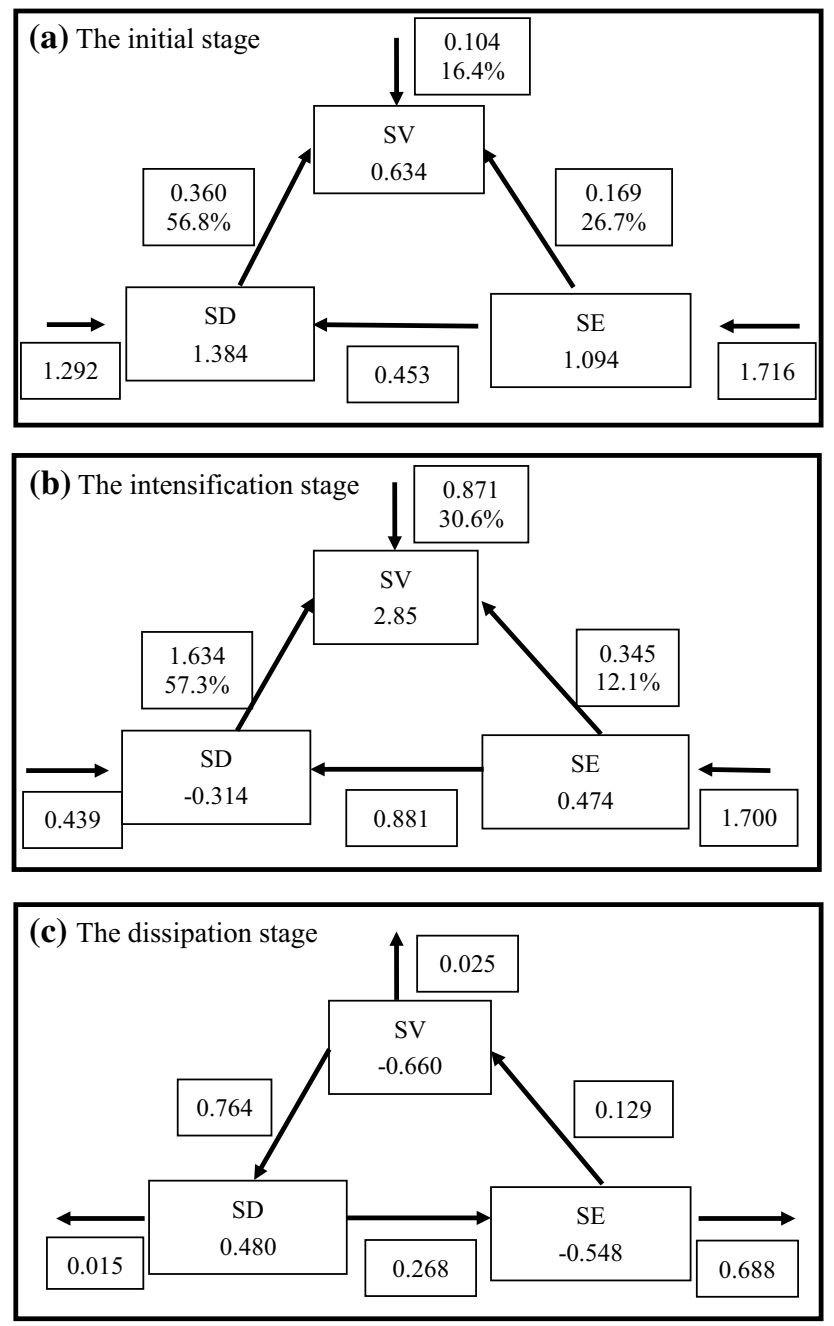

Fig. 7 Area-averaged budgets of SV, SD and SE in the a initiation stage at 1800 UTC 16 August $2009\left(34^{\circ}-36^{\circ} \mathrm{N}, 112^{\circ}-114^{\circ} \mathrm{E}\right)$, b intensification stage at 0600 UTC 17 August $2009\left(33.5^{\circ}-35.5^{\circ} \mathrm{N}\right.$, $114^{\circ}-116^{\circ} \mathrm{E}$ ), and $\mathbf{c}$ dissipation stage at 0600 UTC 18 August 2009 $\left(35^{\circ}-37^{\circ} \mathrm{N}, 118^{\circ}-120^{\circ} \mathrm{E}\right)$ at $800 \mathrm{hPa}$. Units: $10^{-13} \mathrm{~s}^{-2}$

above. The budget values are obtained by averaging the budget terms on the right-hand side of Eqs. (19)-(21) over a small rectangular box around the vortex center (given in Figs. 3 or 6). The use of the rectangular box instead the circular vorticity contours (Schielicke et al. 2016) is on one hand to make the calculation easier and on the other hand is because the average area is mainly around the vortex center and the resolution of the data is only $0.5^{\circ}$, which means the box may contain several data to do the average and this may not make large difference whether one uses the box or the circular. As is shown in Fig. $7 \mathrm{a}$, in the formation stage, all the three types of kinetic quantities show increasing trends. The transformation of SD to SV shows the greatest contribution to the increase in SV, while the residual term of the SD equation is the most important factor for the increase of SD.
The increase of SV and SD simultaneously consumes the deformation, whose development lies in the residual term of the SE equation. In the developing stage (Fig. 7b), the development of SV intensifies evidently, mainly caused by the increase of transformation from SD to SV. Because of the large consumption of divergence by this process, the SD decreases and the transformation of SE to SD becomes the main mechanism maintaining the SD in the developing stage. The SE continues to increase, with a smaller increasing of intensity compared with the formation stage. This is mainly due to the transformation of SE to SD. Compared with the formation stage and developing stage, in the dissipation stage (Fig. 7c), the SV-SD interaction term becomes the deterministic factor for the dissipation of the vortex. The SV-SD interaction term consumes the $\mathrm{SD}$, while the SV-SE interaction term still promotes the development of SV. In addition, the residual terms of the three equations (Eqs. (19)-(21)) all become the consumption mechanisms for the corresponding kinetic quantities, especially the residual term of the SE equation.

\section{Conclusion}

As basic differentiation properties of the air motion, changes of vorticity, divergence and deformation will change the flow pattern, which is an important factor producing the weather. In this paper, the three quantities are studied synchronously instead of just a single quantity. To investigate the interactions of vorticity, divergence and deformation and their roles on the evolution of weather systems, tendency equations of squared vorticity, squared divergence and squared deformation are derived, and the opposite-sign terms between each of the three equations are identified. As the sources or sinks of the corresponding quantities, these opposite-sign terms are considered to control the interactions between vorticity, divergence and deformation.

Through diagnosing the opposite-sign terms in a meso- $\alpha$ low-vortex event in China, the impact of the interactions of vorticity, divergence and deformation on weather system evolution is studied. The results show that the interaction between squared vorticity and squared divergence is the controlling factor for the evolution of the vortex. In the formation stage and rapid intensification stage, squared divergence transforms to squared vorticity to intensify the vortex, and in the dissipation stage the squared vorticity transforms to squared divergence to cause the vortex to die out. This result is consistent with the known mechanism of the role of divergence on generating vorticity, which to some extent verifies the feasibility of our methodology. Apart from this, a new finding is that deformation seems to have played a role in the maintenance of the vortex. In the formation and intensification stage, the squared 
deformation on the one hand transforms to squared vorticity to directly support the increase in vorticity, while on the other hand transforms to squared divergence and contributes to the intensification of the vortex through sustaining the convergence of the flow. In the dissipation stage, squared deformation still transforms to squared vorticity to resist a decrease of vorticity. Meanwhile, the squared divergence begins to transform to squared deformation, which is unfavorable for an increase in divergence but favorable for the maintenance of the vortex. In addition, due to the spatial distribution pattern of the squared vorticity-squared deformation interaction term, i.e. the negative-value area following the positive-value area, deformation also shows an effect on the movement of the vortex.

These results show that the methodology presented in our paper can be useful to study the overall roles of vorticity, divergence and deformation on weather systems evolutions. However, up to now, we have used it for only one case. Different results may appear depending on the scale of the systems. In addition, as stated in Sect. 2, the interactions between vorticity, divergence and deformation are actually related to the change of flow pattern. Therefore, important tasks in the future may include examining the scale dependence of the equations derived in Sect. 2, investigating the role of interactions of the three considered quantities during the transitions of the flow regimes (such as the blocking onset) and also transforming the framework in Sect. 2 to a height coordinate to allow an application to smaller scale systems, such as squall lines.

Acknowledgements This study was supported by the Strategic Pilot Science and Technology Special Program of the Chinese Academy of Sciences (XDA17010105), National Key Research and Development Plan of China (2018YFC1507104), Science and Technology Development Plan Project of Jilin Province (20180201035SF), a project of the National Program on Key Basic Research (91437215), the National Natural Science Foundation of China (41175060, 41575047, 41575065, 41705027 and 4177510), and Open Projects of the Plateau Atmosphere and Environment Key Laboratory of Sichuan Province (PAEKL-2015-K2)
Open Access This article is distributed under the terms of the Creative Commons Attribution 4.0 International License (http://creativeco mmons.org/licenses/by/4.0/), which permits unrestricted use, distribution, and reproduction in any medium, provided you give appropriate credit to the original author(s) and the source, provide a link to the Creative Commons license, and indicate if changes were made.

\section{Appendix}

\section{The differences between vorticity, divergence and deformation}

Figures 8 and 9 present several different types of motions and their effects on an air particle which is a unit cube. OACB is cross section of the air particle in the Oxy surface (Fig. 8a). The velocity of the flow for $O$ has the following three kinds of properties:

1. Pure rotation, no divergence, no deformation:

$\frac{\partial v}{\partial x}=-\frac{\partial u}{\partial y}>0, \quad \frac{\partial u}{\partial x}=\frac{\partial v}{\partial y}=0$.

2. Pure shearing deformation, no rotation, no divergence:

$\frac{\partial v}{\partial x}=\frac{\partial u}{\partial y}>0, \quad \frac{\partial u}{\partial x}=\frac{\partial v}{\partial y}=0$.

3. Rotation + shearing deformation, no divergence

$2 \frac{\partial v}{\partial x}=-\frac{\partial u}{\partial y}>0, \quad \frac{\partial u}{\partial x}=\frac{\partial v}{\partial y}=0$.

The velocities at $A, B$ and $C$ for these three types of flows are, respectively:

$$
\left\{\begin{array} { l } 
{ u _ { A } = u _ { 0 } } \\
{ v _ { A } = v _ { 0 } + ( \frac { \partial v } { \partial x } ) _ { 0 } \delta r }
\end{array} \quad \left\{\begin{array} { l } 
{ u _ { B } = u _ { 0 } + ( \frac { \partial u } { \partial y } ) _ { 0 } \delta r } \\
{ v _ { B } = v _ { 0 } }
\end{array} \quad \left\{\begin{array}{l}
u_{C}=u_{0}+\left(\frac{\partial u}{\partial y}\right)_{0} \delta r \\
v_{C}=v_{0}+\left(\frac{\partial v}{\partial x}\right)_{0} \delta r
\end{array} .\right.\right.\right.
$$


Fig. 8 Schematic diagrams of the change of the air particle for different flows: a the cross section of the air particle in the Oxy surface; b rotation; c shearing deformation and $\mathbf{d}$ rotation and shearing deformation (a)

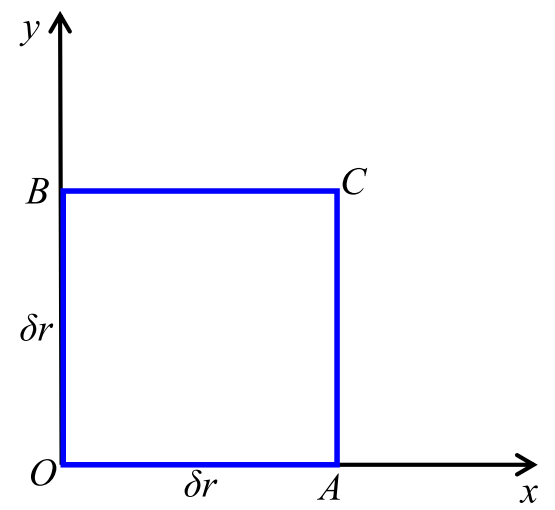

(c)

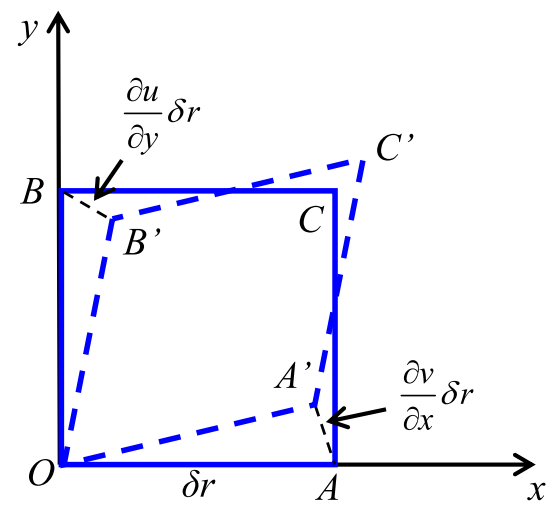

(b)

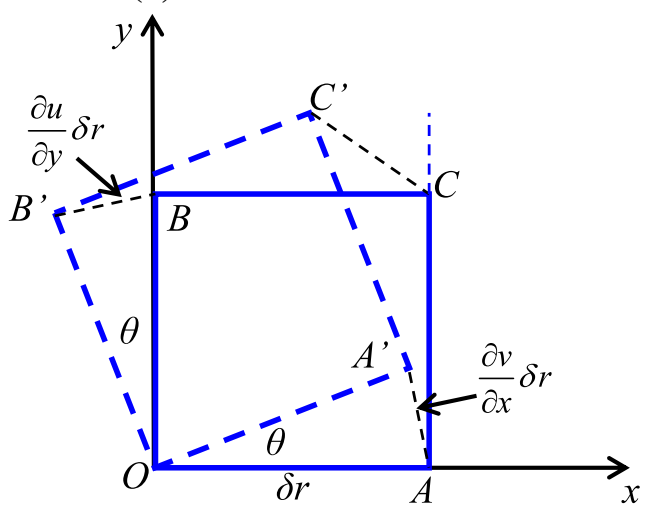

(d)

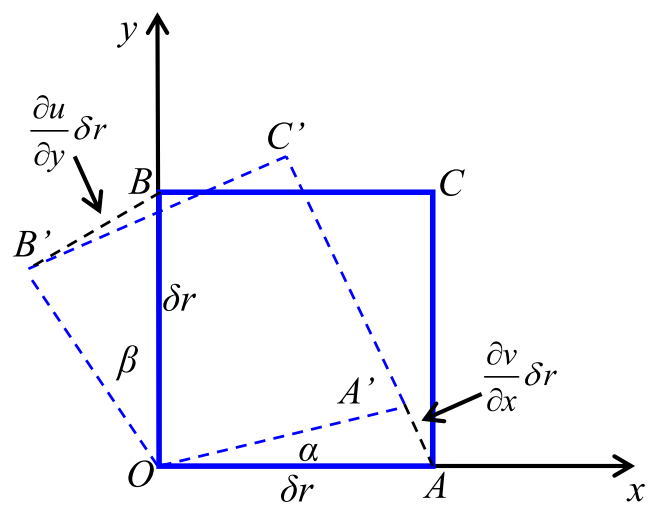

For the pure rotation flow (Eqs. (33) and (36)), under a unit time, as is shown in Fig. $8 \mathrm{~b}$, the point $A$ moves to $A^{\prime}$ through a distance of $(\partial v / \partial x)_{0} \delta r$, meaning that OA rotates to $\mathrm{OA}^{\prime}$ through an angle $\theta=\partial v / \partial x$. Meanwhile, OB rotates to $O B^{\prime}$ through the angle $\theta=-\partial u / \partial y$ (anti-clockwisely) because $\partial u / \partial y=-\partial v / \partial x<0$. Therefore, under this circumstance, $O A C B$ only shows the change of the angle, and the area and shape of $O A C B$ do not change. The rotation speed is measured by vertical vorticity:

$\zeta=\frac{\partial v}{\partial x}-\frac{\partial u}{\partial y}$.

Similarly, under the pure deformation flow (Eqs. (34) and (36)), the square shape of $O A C B$ changes to rhombus, with no rotation or expansion (Fig. 8d). OA and OB rotate towards each other with the angles, respectively, being $\theta=\partial v / \partial x$ (anticlockwise) and $\theta=\partial u / \partial y$ (clockwise). The change of the $O A C B$ is measured by the shearing deformation, namely:

$E_{\mathrm{sh}}=\frac{\partial v}{\partial x}+\frac{\partial u}{\partial y}$.

Figure $8 \mathrm{~d}$ further shows a flow with both deformation and vorticity, and no divergence. According to Eqs. (35) and (36), OA and OB both rotate anti-clockwisely through an angle of $\alpha=\partial v / \partial x$ and $\beta=-\partial u / \partial y$, respectively. Since $\alpha \neq \beta$, both the angle and the shape of $O A C B$ change. This change is measured by both vorticity and deformation:

$\zeta=\frac{\partial v}{\partial x}-\frac{\partial u}{\partial y}=\alpha+\beta$,

$E_{\mathrm{sh}}=\frac{\partial v}{\partial x}+\frac{\partial u}{\partial y}=\alpha-\beta$.

The above two equations show that although according to the definitions of vorticity and deformation, the difference of $\zeta$ and $E_{\mathrm{sh}}$ only shows on the sign of $\partial u / \partial y$, they have very different physical meanings. $\zeta$ is the sum of the rotation angles of $\mathrm{OA}$ and $\mathrm{OB}$, which describes the overall rotation of the unit air particle. $E_{\mathrm{sh}}$ is the difference of the rotation angles of $\mathrm{OA}$ and $\mathrm{OB}$, denoting a relative movement between $\mathrm{OA}$ and $\mathrm{OB}$ and describing a change of the shape of the air particle. The change of the air particle should be described by both vorticity and deformation.

Another group of diagrams to explain the physical meanings of divergence and stretching deformation is given in Fig. 9. The three types of flow are: 
Fig. 9 Schematic diagrams of the change of the air particle for different flows: a divergence;

b) stretching deformation; and (c) divergence and stretching deformation
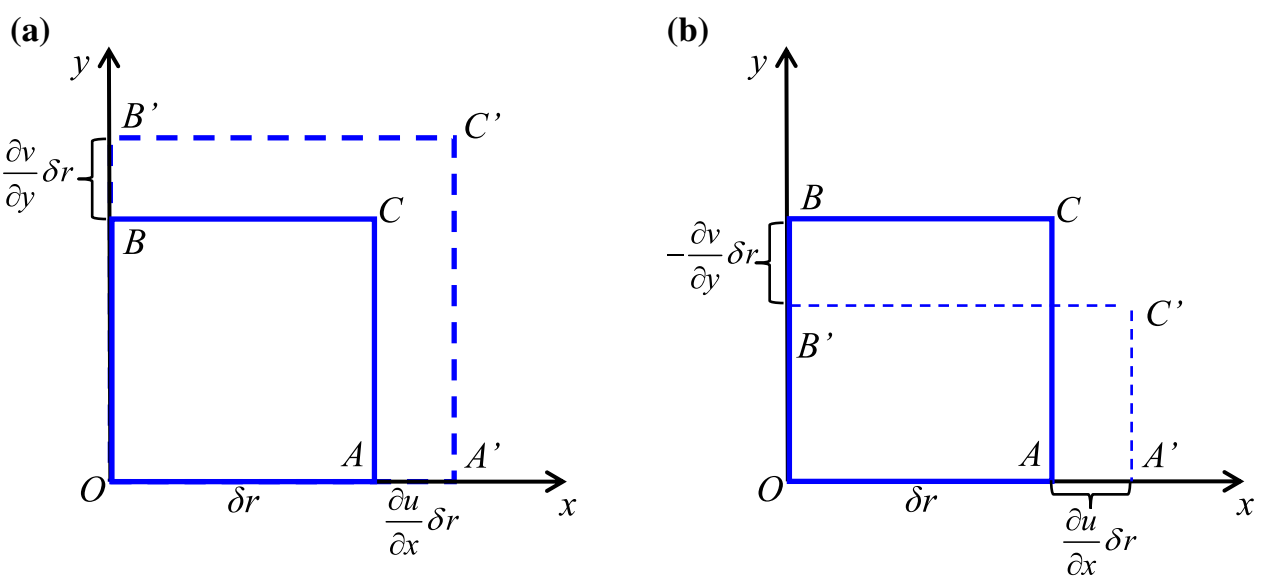

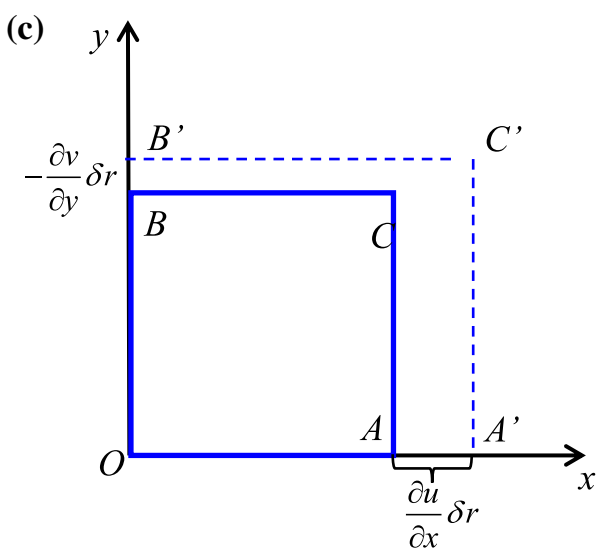

4. Pure divergence, no rotation, no deformation:

$\frac{\partial u}{\partial x}=\frac{\partial v}{\partial y}>0, \quad \frac{\partial v}{\partial x}=\frac{\partial u}{\partial y}=0$

5. Pure stretching deformation, no rotation, no divergence:

$\frac{\partial u}{\partial x}=-\frac{\partial v}{\partial y}>0, \quad \frac{\partial v}{\partial x}=\frac{\partial u}{\partial y}=0$

6. Divergence + stretching deformation, no rotation:

$\frac{\partial u}{\partial x}=2 \frac{\partial v}{\partial y}>0, \quad \frac{\partial v}{\partial x}=\frac{\partial u}{\partial y}=0$

The velocities at $A, B$ and $C$ for these above three types of flows are, respectively:

$\left\{\begin{array}{c}u_{A}=u_{0}+\left(\frac{\partial u}{\partial x}\right)_{0} \delta r \\ v_{A}=v_{0}\end{array} \quad\left\{\begin{array}{l}u_{B}=u_{0} \\ v_{B}=v_{0}+\left(\frac{\partial v}{\partial y}\right)_{0} \delta r\end{array} \quad\left\{\begin{array}{l}u_{C}=u_{0}+\left(\frac{\partial u}{\partial x}\right)_{0} \delta r \\ v_{C}=v_{0}+\left(\frac{\partial v}{\partial y}\right)_{0} \delta r\end{array}\right.\right.\right.$.
As is shown in Fig. 9a, for the pure divergent flow, $O A$ and $O B$ present elongations, respectively, by $\partial u / \partial x \delta r$ and $\partial v / \partial y \delta r$. Therefore, OACB presents expansion of the area, with no change of its angle and shape. The expansion rate is described by divergence:

$D=\frac{\partial u}{\partial x}-\frac{\partial v}{\partial y}$

For the pure stretching flow, OA elongates by $\partial u / \partial x \delta r$ while OB shortens by $-\partial v / \partial y \delta r$. Namely, OACB is stretched in the $x$-direction and contracted in the $y$-direction. Therefore, OACB becomes to a rectangle from a square. The stretching rate is described by the stretching deformation:

$E_{\mathrm{st}}=\frac{\partial u}{\partial x}+\frac{\partial v}{\partial y}$

The difference between stretching deformation and divergence can be more easily understood with Fig. 9c, which 
shows the change of air particle with both deformation and divergence. According to Eqs. (43) and (44), OA and OB present elongations, respectively, by $m=\partial u / \partial x \delta r$ and $n=\partial v / \partial y \delta r$. Since $m \neq n$, both the area and the shape of OACB change. This change is measured by divergence and stretching deformation:

$D=\frac{\partial u}{\partial x}+\frac{\partial v}{\partial y}=m+n$,

$E_{\mathrm{st}}=\frac{\partial u}{\partial x}-\frac{\partial v}{\partial y}=m-n$.

From the above two equations, one can find the very different physical meanings between $D$ and $E_{\mathrm{st}} . D$ is the sum of the elongation distances of $\mathrm{OA}$ and $\mathrm{OB}$, which describe the expansion of the unit air particle. $E_{\mathrm{st}}$ is the difference of the elongation distances of $\mathrm{OA}$ and $\mathrm{OB}$, denoting a relative change between $\mathrm{OA}$ and $\mathrm{OB}$. Therefore, $E_{\mathrm{st}}$ describes the change of the shape of the air particle.

The above analysis shows that for the velocity itself, the deformational motion is mostly part of the rotational motion and the divergent motion which are inverted from vorticity and divergence. Deformation has very different physical meanings to vorticity and divergence. For any small particles in the fluid, deformation, together with vorticity and divergence, are the basic factors of the everchanging atmosphere and the weather formation. From this attitude, we think that SE is not a part of SV and a part of SD.

\section{References}

Bishop CH (1996a) Domain-Independent attribution. Part I: reconstructing the wind from estimates of vorticity and divergence using free space green's functions. J Atmos Sci 53:241-252

Bishop CH (1996b) Domain-Independent attribution. Part II: its value in the verification of dynamical theories of frontal waves and frontogenesis. J Atmos Sci 53:253-262

Bluestein HB (1992) Synoptic-dynamic meteorology in midlatitudes. Oxford University Press, New York

Cai M (1992) A physical interpretation for the stability property of a localized disturbance in a deformation flow. J Atmos Sci 49:2177-2182

Cao J, Xu Q (2011) Computing streamfunction and velocity potential in a limited domain of arbitrary shape. Part II: numerical methods and test experiments. Adv Atmos Sci 28:1445-1458

Cao J, Sun J, Gao S, Cui X (2013) Kinetic energy budget equations of rotational and divergent flow in terrain-following coordinates. Atmos Ocean Sci Lett 6:149-153

Cao J, Ran L, Li N (2014) An application of the Helmholtz theorem in extracting the externally induced deformation field from the total wind field in a limited domain. Mon Weather Rev 142:2060-2066
Chen QS, Kuo YH (1992a) A Harmonic-Sine series expansion and its application to partitioning and reconstruction problems in a limited area. Mon Weather Rev 120:91-112

Chen QS, Kuo YH (1992b) A consistency condition for the wind field reconstruction in a limited area and a harmonic-cosine series expansion. Mon Weather Rev 120:2653-2670

Danielsen EF (1968) Stratospheric-tropospheric exchange based on radioactivity, ozone and potential vorticity. J Atmos Sci 25:502-518

Davis CA, Emanuel KA (1991) Potential vorticity diagnostics of cyclogenesis. Mon Weather Rev 119:1929-1953

Ding Y, Liu Y (1985) On the analysis of typhoon kinetic energy, Part II: conversion between divergent and nondivergent wind. Sci China 15(11):1045-1054

Dutton JA (1976) The Ceaseless wind: an introduction to the theory of atmospheric motion. McGraw-Hill, New York

Fu S, Sun J, Zhao S, Li W (2011) The energy budget of a southwest vortex with heavy rainfall over South China. Adv Atmos Sci 20:638-649

Gao ST (2007) Dynamics and forecasting methods of the mesoscale motions in atmosphere. Meteorological Press, Beijing

Haynes PH, McIntyre ME (1987) On the evolution of vorticity and potential vorticity in the presence of diabatic heating and frictional or other forces. J Atmos Sci 44:828-841

Holton JR (2004) An introduction to dynamic meteorology. Elsevier Academic Press, New York

Hoskins BJ, Hodges KI (2002) New perspectives on the Northern Hemisphere winter storm tracks. J Atmos Sci 59(6):1041-1061

Hoskins BJ, McIntyre ME, Robertson AW (1985) On the use and significance of isentropic potential vorticity maps. Q J R Meteorol Soc 111:877-946

Kalthoff N, Adler B, Barthlott CH et al (2009) The impact of convergence zones on the initiation of deep convection: a case study from COPS. Atmos Res 93:680-694

Keyser D, Pecnick MJ (1985) A two-dimensional primitive equation model of frontogenesis forced by confluence and horizontal shear. J Atmos Sci 42(12):1259-1282

Keyser D, Reeder MJ, Reed RJ (1988) A generalization of Petterssen's frontogenesis function and its relation to the forcing of vertical motion. Mon Weather Rev 116:762-780

Krishnamurti TN, Ramanathan Y (1982) Sensitivity of the monsoon onset to differential heating. J Atmos Sci 39:1290-1306

Luo ZX, Dai K (2008) A climatological investigation of the activity of summer subtropical vortices. Acta Meteorol Sin 22(1):1-7

Lv MY, Hou ZM, Zhou Y (2008) Dynamic meteorology. China Meteorological Press, Beijing, pp 122-124

Mak M, Cai M (1989) Local barotropic instability. J Atmos Sci 46:3289-3331

Masunaga H (2013) A satellite study of tropical moist convection and environment variability: a moisture and thermal budget analysis. J Atmos Sci 70:2443-2446

Montgomery MT, Enagonio J (1998) Tropical cyclogenesis via convectively forced vortex rossby waves in a three-dimensional quasigeostrophic model. J Atmos Sci 55:3176-3207

Okubo A (1970) Horizontal dispersion of floatable particles in the vicinity of velocity singularity such as convergences. Deep-Sea Res 17:445-454

Olsson PQ, Cotton WR (1997) Balanced and unbalanced circulations in a primitive equation simulation of a midlatitude MCC. Part II: analysis of balance. J Atmos Sci 54:479-497

Petterssen S (1956) Motion and motion systems. Weather analysis and forecasting. McGraw-Hill, New York, pp 1-428

Raymond DJ (2006) Nonlinear balance and potential-vorticity thinking at large Rossby number. Q J R Meteorol Soc 118:987-1015

Raymond DJ, Jiang H (1990) A theory for long-lived mesoscale convective systems. J Atmos Sci 47:3067-3077 
Rozoff CM, Schubert WH, McNoldy BD, Kossin JP (2006) Rapid filamentation zones in intense tropical cyclones. J Atmos Sci 63:325-340

Sadarjoen LA, Post FH (2000) Detection, quantification, and tracking of vortices using streamline geometry. Comput Graph 24:333-341

Schielicke L, Nevir P, Ulbrich U (2016) Kinematic vorticity number-a tool for estimating vortex sizes and circulations. Tellus A 68:29464

Spensberger C, Spengler T (2014) A new look at deformation as a diagnostic for large-scale flow. J Atmos Sci 71:4221-4234

Stevens DE (1979) Vorticity, momentum and divergence budgets of synoptic-scale wave disturbances in the tropical Eastern Atlantic. Mon Weather Rev 107:535-550

Stone PH (1966) Frontogenesis by horizontal wind deformation fields. J Atmos Sci 23:455-465

Tsai YM, Kuo HC, Schubert WH (2010) Filamentation time diagnosis of thinning troughs and cutoff lows. Mon Wea Rev 138:2327-2335

Uccellini LW, Koch SE (1987) The synoptic setting and possible energy sources for musical wave disturbances. Mon Weather Rev 115:721-729

Ueno K, Takano S, Kusaka H (2009) Nighttime precipitation induced by a synoptic-scale convergence in the Central Tibetan Plateau. J Meteor Soc Jpn 87:459-472
Wang Y (2008) Rapid filamentation zone in a numerically simulated tropical cyclone. J Atmos Sci 65:1158-1181

Wang Z, Sun S (1988) The relationship between environmental vorticity and divergence field associated with heavy rain systems. Acta Meteor Sinica 46:492-496

Weiss J (1991) The dynamics of enstrophy transfer in two-dimensional hydrodynamics. Phys D 48:273-294

Yang X, Jiang J, Hu S (2013) Analysis on an excessive precipitation process triggered by warm shear line with low vortex in southern Shandong on 17 August 2009. Torrential Rain Disasters 32:224-234

Zhang FQ (2004) Generation of mesoscale gravity waves in uppertropospheric jet-front systems. J Atmos Sci 61:440-457

Zhou YS, Cao J (2010) Partitioning and reconstruction problem of the wind in a limited region. Acta Phys Sin 59:2898-2906

Ziegler C, Tsengdar JL, Roger APS (1997) Convective initiation at the dryline: a modeling study. Mon Weather Rev 125:1001-1026

Publisher's Note Springer Nature remains neutral with regard to jurisdictional claims in published maps and institutional affiliations. 